International Journal of Bifurcation and Chaos, Vol. 12, No. 11 (2002) 2501-2522

(c) World Scientific Publishing Company

\title{
ROBUST HETEROCLINIC CYCLES IN TWO-DIMENSIONAL RAYLEIGH-BÉNARD CONVECTION WITHOUT BOUSSINESQ SYMMETRY
}

\author{
ISABEL MERCADER \\ Departament de Física Aplicada, Universitat Politècnica de Catalunya, Barcelona, Spain \\ JOANA PRAT \\ Departament de Matemàtica Aplicada IV, \\ Universitat Politècnica de Catalunya, Barcelona, Spain \\ EDGAR KNOBLOCH \\ Department of Applied Mathematics, University of Leeds, Leeds LS2 9JT, UK
}

Received October 31, 2001; Revised March 26, 2002

\begin{abstract}
The onset of convection in systems that are heated via current dissipation in the lower boundary or that lose heat from the top boundary via Newton's law of cooling is formulated as a bifurcation problem. The Rayleigh number as usually defined is shown to be inappropriate as a bifurcation parameter since the temperature difference across the layer depends on the amplitude of convection and hence changes as convection evolves at fixed external parameter values. A modified Rayleigh number is introduced that does remain constant even when the system is evolving, and solutions obtained with the standard formulation are compared with those obtained via the new one. Near the 1:2 spatial resonance in low Prandtl number fluids these effects open up intervals of Rayleigh number with no stable solutions in the form of steady convection or steadily traveling waves. Direct numerical simulations in two dimensions show that in such intervals the dynamics typically take the form of a nearly heteroclinic modulated traveling wave. This wave may be quasiperiodic or chaotic.
\end{abstract}

Keywords: Convection; mode interactions; heteroclinic cycles.

\section{Introduction}

Rayleigh-Bénard convection has been the subject of a large number of theoretical and experimental studies since the pioneering work of Lord Rayleigh and H. Bénard. Yet despite this there are still various issues whose significance has not been fully recognized. One such issue that is important for the interpretation of experiments relates to the proper boundary conditions to be used in any theoretical treatment of the problem. In papers in which comparison between experiment and theory is attempted this important question is usually dismissed with the claim that boundaries consisting of a "good" thermal conductor correspond to fixed temperature boundary conditions while those made of a thermal insulator correspond to fixed heat flux. For example, it is commonly believed that since copper is a good thermal conductor, copper boundaries are always correctly modeled by constant temperature boundary conditions. However, the situation is not so simple. Whether or not a boundary behaves like a constant temperature boundary does not depend only on its composition but also on its heat capacity relative to the heat capacity of the fluid with which it is in contact. The basic issue is fundamentally whether a change in the temperature of the fluid in contact with the boundary produces a 
small change in the temperature of the boundary or a large one. If it is the former then the boundary is effectively a constant temperature one; otherwise it is not. Both the conductivity of the material of the boundary (and of the confined fluid) and its mass enter into the determination of the (dimensionless) Biot number that characterizes the thermal properties of the boundary when in contact with a given mass of fluid. Regrettably, experimentalists almost never provide sufficient information to allow a theorist to estimate the Biot numbers for the lower and upper boundaries used in their experiment. It is this fact that is responsible for one of the main difficulties in comparing experiment with theory.

These issues are particularly acute when one studies problems in which the bifurcation to convection is subcritical. In this case once the conduction state loses stability the system evolves far from the initial state and the temperature difference $\Delta T$ across the layer drops, since convection decreases the temperature of the lower boundary and increases that of the upper boundary. In a bifurcation diagram showing the Nusselt number (a dimensionless measure of the heat transport) against the conventionally defined Rayleigh number (proportional to $\Delta T$ ) the system therefore follows a path that slopes to the left, instead of evolving vertically as one would expect of a system under constant conditions. This behavior is seen quite dramatically in a number of the early experiments on binary fluid convection in which the lower boundary was heated electrically, via constant electrical power [Surko et al., 1986; Sullivan \& Ahlers, 1988; Steinberg et al., 1989], indicating that for these experiments the assumption that the lower boundary is a fixed temperature boundary is in fact incorrect, a fact noted already by Villers and Platten [1984].

In this article we indicate how the convection problem can be reformulated in order to define a bifurcation parameter that remains constant under fixed external conditions, and focus on the case in which the Biot numbers of the upper and lower boundaries are distinct. The use of the Biot numbers is advantageous because it supplants the solution of the time-dependent conduction problem in boundaries of finite width [Proctor, 1981; Recktenwald \& Lücke, 1998], subject to a fixed temperature outer boundary condition if the boundary is held in thermal contact with a heat bath at fixed temperature. Note that the notion of a heat bath requires that the thermal capacity of the bath be large compared to that of the sample. Whether or not a boundary acts like a constant temperature heat bath under experimental conditions depends therefore on its heat capacity, and a boundary composed of a good thermal conductor may still be unable to smooth out temperature fluctuations in the adjacent fluid.

We assume here that the fluid is Boussinesq so that its properties (with the exception of the density) may be taken to be constant, and use noslip boundary conditions at the top and bottom. The resulting system has Boussinesq symmetry if the Biot numbers at the top and bottom are likewise identical. In earlier work [Prat et al., 1998] we showed, following Armbruster [1987], that even two-dimensional convection is strongly affected by the presence of Boussinesq symmetry. This is because Boussinesq symmetry changes the relative importance of certain spatial resonances. In particular the Boussinesq symmetry turns the $1: 2$ resonance into a weak resonance but has no effect on the $1: 3$ resonance. As a result the moderate amplitude behavior of systems with Boussinesq symmetry is dominated by the $n_{1}: n_{2}=1: 3$ spatial resonance while the $1: 2$ resonance remains the dominant resonance in systems without this symmetry. At a $n_{1}: n_{2}$ spatial resonance the conduction state of the system is marginally stable with respect to two spatial modes with distinct wavenumbers, of the form $2 \pi n / L$, where $n$ is an integer and $L$ is the imposed spatial period in the horizontal. Since the dynamics near the $1: 2$ and $1: 3$ resonances are quite different [Porter \& Knobloch, 2000, 2001] it is of interest to explore the crossover from one type of behavior to the other, particularly in low Prandtl number fluids since low Prandtl numbers favor dynamics. In previous papers we have used numerical branch following techniques to explore the behavior near the $1: 3$ resonance in systems with Boussinesq symmetry [Prat et al., 1998], and to investigate in detail how the $1: 2$ resonance comes to dominate the dynamics as the Boussinesq symmetry is progressively broken [Mercader et al., 2001; Prat et al., 2002]. We accomplished this by homotopically continuing the velocity boundary conditions at the top from no-slip to free-slip, while keeping the lower boundary no-slip, and discovered that the progressive loss of symmetry opens up intervals in Rayleigh number in which none of the simple solutions known to be present near the $1: 2$ mode interaction (the $n=1$ and $n=2$ steady states, and traveling waves) is stable. As shown below, the results are similar if the velocity boundary conditions at top and 
bottom remain no-slip but the top layer radiates heat to the outside via Newton's law of cooling while the bottom temperature remains fixed, or if the bottom plate is heated electrically via constant current dissipation while the temperature at the top remains fixed. In these interesting Rayleigh number intervals complex dynamical behavior may be expected. We report here the results of direct numerical simulations of the governing equations in two dimensions, and show that the dynamics in these regimes may take the form of either quasiperiodic modulated traveling waves or one of two types of (nearly) heteroclinic traveling waves that may be periodic or chaotic, depending on initial conditions. Similar behavior occurs in layers that are heated with constant electrical power, modeled here using a finite Biot number lower boundary.

\section{Modified Rayleigh Number}

We consider Boussinesq convection between boundaries responsible for the boundary conditions

$$
\begin{array}{ll}
\frac{d T_{-}}{d z}=-\frac{B_{-}}{d}\left(T_{L}-T_{-}\right) & \text {at } z=-\frac{d}{2}, \\
\frac{d T_{+}}{d z}=-\frac{B_{+}}{d}\left(T_{+}-T_{U}\right) & \text { at } z=\frac{d}{2},
\end{array}
$$

where $d$ is the layer depth, $T_{L}, T_{U}$ are the temperatures of the lower (L) and upper (U) heat baths, and $T_{-}, T_{+}$are the temperatures in the fluid right next to the lower and upper boundaries (see Fig. 1). Here $B_{\mp}$ are the Biot numbers of the boundaries: perfectly conducting boundaries correspond to $B=\infty$ while an insulating boundary corresponds to $B=0$. In the following we assume that the Biot numbers are constants, independent of the wavelength of the pattern. This is so whenever the wavelength exceeds the thickness of the boundary plates [Platten

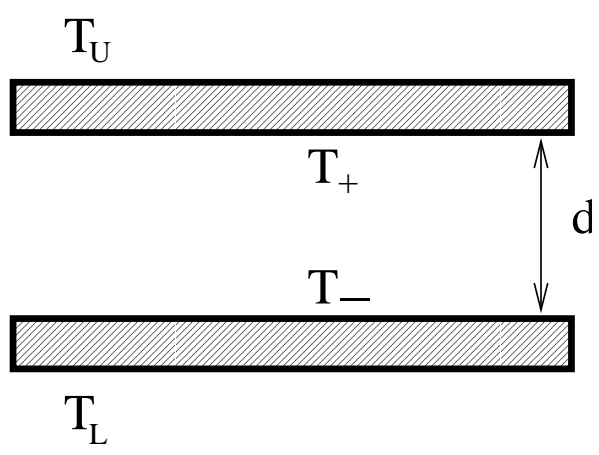

Fig. 1. Sketch of the fluid layer.
\& Legros, 1984]. Note that, by hypothesis, $T_{L}, T_{U}$ are independent of time, while $T_{-}, T_{+}$fluctuate in response to the motion of the fluid.

In the standard description of the RayleighBénard problem one describes the system in terms of the dimensionless temperature difference across the fluid in the conduction state, regardless of whether this state is stable or not. This is a convenient way of describing the system because in the presence of perfectly thermally conducting boundaries this temperature difference remains constant even after instability has set in. More generally, the temperature in the conduction state satisfies an equation of the form $d T / d z=-\Delta T^{c} / d$, and so

$$
\Delta T^{c}=B_{-}\left(T_{L}-T_{-}^{c}\right)=B_{+}\left(T_{+}^{c}-T_{U}\right) .
$$

Here $T_{\mp}^{c}$ are the temperatures at the bottom and top of the fluid in the conduction state. Since $T_{-}^{c}-T_{+}^{c}=\Delta T^{c}$ it follows that

$$
\Delta T^{c}=\left(T_{L}-T_{U}\right) \frac{B_{+} B_{-}}{B_{+} B_{-}+B_{+}+B_{-}},
$$

and is therefore constant provided $T_{L}-T_{U}$ remains fixed. Moreover, in view of the equivalent relation,

$$
\Delta T^{c}=\frac{B_{+} B_{-} \Delta T-d\left(B_{+} T_{-}^{\prime}+B_{-} T_{+}^{\prime}\right)}{B_{+} B_{-}+B_{+}+B_{-}},
$$

we see that the temperature difference $\Delta T \equiv T_{-}-$ $T_{+}$across the fluid may indeed change during evolution but must be compensated by a corresponding variation in the vertical temperature gradients (indicated by a prime) at the top and bottom. We propose therefore to define a modified Rayleigh number based on the quantity $\Delta T^{c}$ (as opposed to $\Delta T)$ as a proper bifurcation parameter for the system (cf. Prat et al. [2001]).

To do this we nondimensionalize the equations in the usual way, by expressing the temperature $T$ in units of $\Delta T^{c}$, distances in units of the layer depth $d$ and time in units of $d^{2} / \kappa$, where $\kappa$ is the thermal diffusivity of the fluid. We define the dimensionless control parameter by the relation [Clune, 1993]

$$
\mathrm{Ra}^{\prime \prime}=\frac{\alpha g\left(T_{L}-T_{U}\right) d^{3}}{\kappa \nu}=\mathrm{Ra}^{\prime} \frac{T_{L}-T_{U}}{\Delta T^{c}},
$$

where $\mathrm{Ra}^{\prime}$ is defined by analogy to the usual Rayleigh number, i.e. $\mathrm{Ra}^{\prime}$ is proportional to the temperature difference $\Delta T^{c}$ across the fluid in the conduction state, and so

$$
\mathrm{Ra}^{\prime}=\frac{\alpha g d^{3}}{\kappa \nu} \frac{B_{+} B_{-} \Delta T-d\left(B_{+} T_{-}^{\prime}+B_{-} T_{+}^{\prime}\right)}{B_{+} B_{-}+B_{+}+B_{-}} .
$$


Note that both $\mathrm{Ra}^{\prime}$ and $\mathrm{Ra}^{\prime \prime}$ defined in this manner remain constant for fixed external conditions. Thus both provide good definitions of a control parameter, in contrast to the conventionally defined Rayleigh number

$$
\mathrm{Ra}=\frac{\alpha g \Delta T d^{3}}{\kappa \nu} .
$$

However, $\mathrm{Ra}^{\prime}$ reduces to $\mathrm{Ra}$ in the limit of infinitely conducting boundaries $\left(B_{ \pm} \rightarrow \infty\right)$, as it must. Note that the Rayleigh number $\mathrm{Ra}^{\prime \prime}$ is defined in terms of $T_{L}-T_{U}$ and not the temperature difference across the fluid, and hence is in principle easily measured in any experiment.

In the following we list the final dimensionless equations describing convection in two dimensions with periodic boundary conditions in the horizontal $(x)$ direction, expressing the temperature fluctuation $\theta$ away from the conduction profile $T_{-}^{c}-$ $\Delta T^{c}(z / d+1 / 2)$ in units of $\Delta T^{c}$. These are written in terms of a mean flow $\mathbf{U}=(U, 0)$ and its fluctuating part $\mathbf{v}^{\prime}=\left(-\partial_{z} \chi^{\prime}, \partial_{x} \chi^{\prime}\right)$, where $\overline{\mathbf{v}}^{\prime}=\overline{\chi^{\prime}}=0$, with the overline indicating an average over the horizontal period [Prat et al., 1995]. The result is

$$
\begin{gathered}
\left(\partial_{t}-\sigma \partial_{z z}^{2}\right) U+\partial_{z} \overline{v_{x}^{\prime} v_{z}^{\prime}}=0 \\
\left(\partial_{t}+U \partial_{x}-\sigma \nabla^{2}\right) \omega^{\prime}+\operatorname{Ra}^{\prime} \sigma \partial_{x} \theta+\partial_{z z}^{2} U \partial_{x} \chi^{\prime} \\
+\frac{\partial\left(\chi^{\prime}, \omega^{\prime}\right)}{\partial(x, z)}-\frac{\overline{\partial\left(\chi^{\prime}, \omega^{\prime}\right)}}{\partial(x, z)}=0 \\
\left(\partial_{t}+U \partial_{x}-\nabla^{2}\right) \theta-\partial_{x} \chi^{\prime}+\frac{\partial\left(\chi^{\prime}, \theta\right)}{\partial(x, z)}=0
\end{gathered}
$$

where $\omega^{\prime}=-\nabla^{2} \chi^{\prime}$, and $\sigma$ is the Prandtl number. Note that $\operatorname{Ra}=\operatorname{Ra}^{\prime}\left(1+\bar{\theta}_{-}-\bar{\theta}_{+}\right)$.

The boundary conditions on the temperature fluctuation $\theta$ are

$$
\left(1-B_{ \pm}^{*}\right) \partial_{z} \theta=\mp B_{ \pm}^{*} \theta \quad \text { at } z= \pm \frac{1}{2}
$$

where $B_{ \pm}^{*}=B_{ \pm} /\left(1+B_{ \pm}\right)$. The modified Biot numbers $B_{ \pm}^{*}$ are convenient for numerical exploration, and are such that $B=0(\infty)$ corresponds to $B^{*}=0(1)$. For no-slip boundaries the velocity boundary conditions are

$$
U=\chi^{\prime}=\partial_{z} \chi^{\prime}=0 \quad \text { at } z= \pm \frac{1}{2} .
$$

Note that the boundary conditions on the temperature perturbation $\theta$ involve the Biot numbers and hence differ from the standard situation. As a result the steady states with $B_{ \pm}<\infty$ differ from those with perfectly conducting boundary conditions (cf. Fig. 2). In the case $B_{ \pm} \ll 1$ the primary instability sets in with a small wavenumber, and a single evolution equation for the horizontal planform can be derived ([Knobloch, 1990; Petrescu, 1998], and references therein). In one dimension this equation exhibits behavior related to the presence of the $1: 2$ spatial resonance including the associated structurally stable heteroclinic cycles present for sufficiently small Prandtl numbers [Cox, 1996]; for infinite Prandtl number a related equation describes a variety of twodimensional patterns of large spatial scale and the transitions among them [Knobloch, 1990; Pontes et al., 1996, 1999], while for finite Prandtl numbers the long wave vertical vorticity mode [Pismen, 1986] leads to yet richer behavior [Petrescu, 1998].

Figure 2 shows the results obtained by solving Eqs. $(2 \mathrm{a})-(2 \mathrm{e})$ for the case $\left(B_{-}^{*}, B_{+}^{*}\right)=(1,0.8)$ and $\sigma=0.1$ using a spectral Galerkin-Fourier technique in $x$ and collocation-Chebyshev in $z$ [Prat et al., 1998; Drissi et al., 1999] in a periodic box of $L=\pi$ (i.e. $k \equiv 2 \pi / L=2$ ). For these parameters the $1: 2$ interaction occurs at $\mathrm{Ra}^{\prime}=1786.03, k=2.04$, and the Boussinesq symmetry is broken. Here and elsewhere we use $N-1$ as a measure of the amplitude of convection, where $N$ (the Nusselt number) denotes the total heat flux across the layer measured in units of the conductive heat flux, the latter calculated using the temperature difference $\Delta T^{c}$ defined above. As a result the state $N=1$ corresponds to the pure conduction state, with no motion present. The figure shows $N-1$ for the $n=1,2$ convective states (solid curves) as a function of (a) $\mathrm{Ra}^{\prime}$ and (b) Ra, and compares the results with those for the $n=2$ state when $B_{-}^{*}=B_{+}^{*}=1$ (dotted curve). For these parameter values $(2 \pi / L<2.04)$ the $n=2$ state bifurcates from the conduction state before $n=1$ state and does so supercritically.

The evolution from the unstable conduction state that results is indicated by arrows. These are vertical in Fig. 2(a) but slope to the left as in Fig. 2(b) whenever $B_{+}^{*}<1$. This slope can be used as a diagnostic for the value of the Biot number. In both cases the Nusselt number in the final state is independent of $z$ and hence equals the Nusselt number $N_{+}$at the top; since the convective flux vanishes at the boundary the latter is given (in terms of dimensional variables) by $N_{+} \equiv-d \partial_{z} \bar{T}_{+} / \Delta T^{c}$. Using the boundary condition at the top one finds 


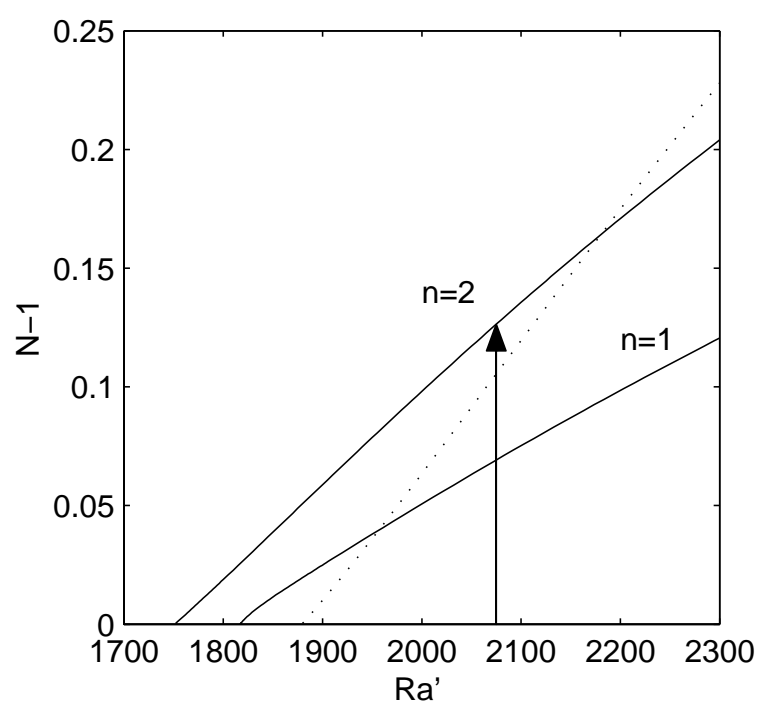

(a)

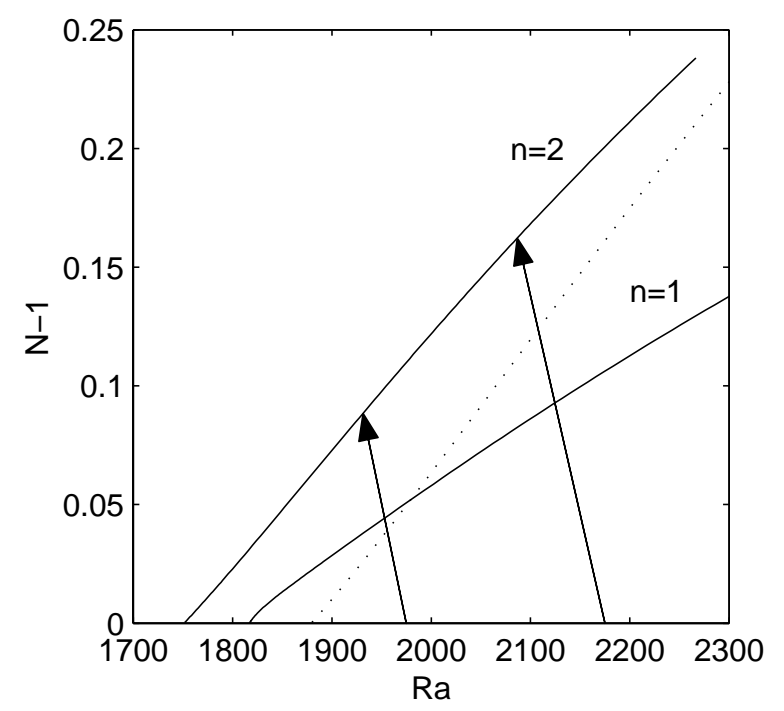

(b)

Fig. 2. The Nusselt number $N$ for the system (2a)-(2e) with $\sigma=0.1, B_{+}^{*}=0.8, B_{-}^{*}=1$ and $k=2$ as a function of (a) $\mathrm{Ra}^{\prime}$ and (b) Ra. The primary instability is to an $n=2$ mode (at $\mathrm{Ra}^{\prime}=1751.23$ or equivalently $\mathrm{Ra}^{\prime \prime}=2187$ ), followed by an instability to an $n=1$ mode at $\mathrm{Ra}^{\prime}=1816.87\left(\mathrm{Ra}^{\prime \prime}=2268\right)$. The dotted line shows $n=2$ branch for $B_{+}^{*}=B_{-}^{*}=1$ and is identical in both plots. The arrows connect initial states with final states, showing that the conventionally defined Rayleigh number Ra decreases during evolution (b); this is not the case in (a).

that $N_{+}=B_{+}\left(\bar{T}_{+}-T_{U}\right) / \Delta T^{c}$, where $T_{+}$is the temperature of the fluid next to the boundary; in a convecting state this temperature is, in general, greater than $T_{+}^{c}$, the temperature in the conduction state, because the convection transports heat upwards from the lower boundary. For a perfect conductor at the bottom one finds

$$
N_{+}-1=\frac{B_{+}^{*}}{1-B_{+}^{*}} \frac{\mathrm{Ra}^{\prime}-\mathrm{Ra}}{\mathrm{Ra}^{\prime}}
$$

Thus, provided $B_{+}^{*}>0$, we may use either $N_{+}-1$ or $\left(\mathrm{Ra}^{\prime}-\mathrm{Ra}\right) / \mathrm{Ra}^{\prime}$ as indicators of the amplitude of the convection that develops from an unstable conduction state once $\mathrm{Ra}^{\prime}$ exceeds the critical value for convective instability. In the special case of a perfectly insulating boundary, $B_{+}^{*}=0$, the Nusselt number $N_{+}=N_{-}=1$ for all steady or steadily translating states. This is so also whenever convection is driven by constant heat flux $\dot{Q}$ at the bottom (Sec. 3.2). In this case $B_{-}^{*}=0$, and we define $\Delta T^{c}=\dot{Q} d / K$, where $K$ is the thermal conductivity. In either of these cases we use the quantity $\left(\mathrm{Ra}^{\prime}-\mathrm{Ra}\right) / \mathrm{Ra}^{\prime}$ (or equivalently $\left.\left(\mathrm{Ra}^{\prime}-\mathrm{Ra}\right) / \mathrm{Ra}\right)$ as a measure of the solution amplitude.

We emphasize that the solid and dotted curves in Fig. 2 for $n=2$ states are not the same, i.e. the assertion that Biot numbers only affect the evolutionary path in the Nusselt number-Rayleigh number diagram ( $N$-Ra diagram) but not the final equilibrated state is manifestly false.

\section{Nearly Heteroclinic Cycles}

In this section we summarize our results on nearly heteroclinic cycles in the interesting case $B_{+}^{*} B_{-}^{*}<1$ and unequal Biot numbers. We consider two cases, (i) $B_{+}^{*}=0.8, B_{-}^{*}=1$, corresponding to a perfectly thermally conducting lower boundary, and heat loss from the upper boundary, and (ii) $B_{+}^{*}=1, B_{-}^{*}=0$, corresponding to a system heated via constant heat flux through the lower boundary, with a perfectly thermally conducting upper boundary. These examples illustrate the two distinct mechanisms identified in normal form theory that produce attracting structurally stable heteroclinic cycles.

\section{1. $B_{+}^{*}=0.8, B_{-}^{*}=1$}

For these values of the Biot numbers Fig. 2 shows that nothing interesting occurs for $k<2.04$. However, this is no longer so when $k>2.04$. Figure 3 shows the Nusselt number $N$ as a function of $\mathrm{Ra}^{\prime}$ obtained from Eqs. (2a)-(2e) for $\sigma=0.1$ and $k=2.08$. The figure shows that the branch of steady states with $n=1$ now bifurcates from the conduction state before the pure modes $n=2$. The 

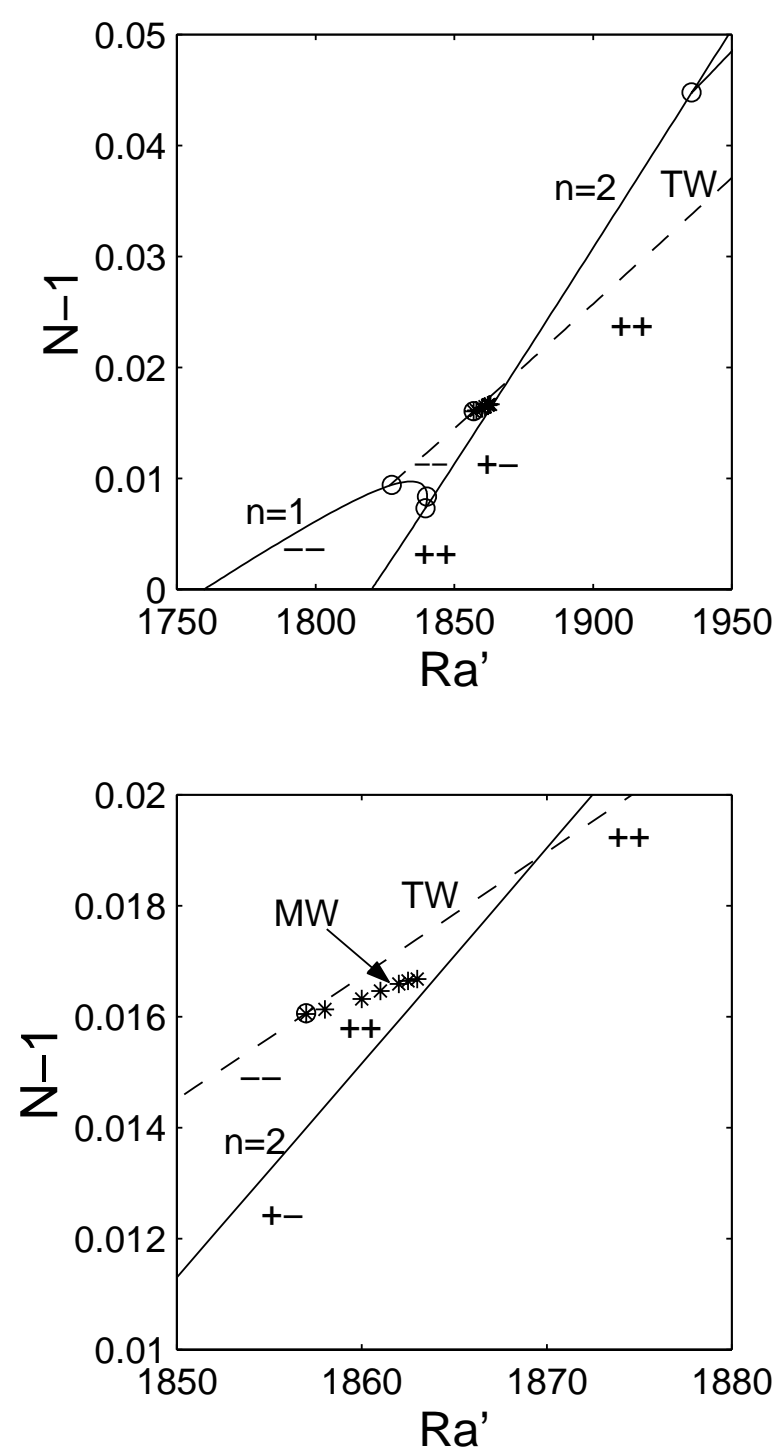

Fig. 3. Time-averaged Nusselt number $N$ as a function of $\mathrm{Ra}^{\prime}$ for $\sigma=0.1, B_{+}^{*}=0.8, B_{-}^{*}=1$ and $k=2.08$, showing steady states $(-)$, TW (--) and MW (*). Open circles denote local bifurcations.

$n=1$ branch is initially stable but loses stability as $\mathrm{Ra}^{\prime}$ increases at a parity-breaking bifurcation at $\mathrm{Ra}^{\prime} \approx 1826$ that produces a pair of traveling waves (dashed line), one traveling to the left and the other to the right. These traveling waves (hereafter TW) bifurcate supercritically and hence are initially stable. The (now unstable) $n=1$ branch undergoes a saddle-node bifurcation $\left(\mathrm{Ra}^{\prime}=1840.1\right)$ before terminating on the $n=2$ branch in a steady state bifurcation $\left(\mathrm{Ra}^{\prime}=1839.6\right)$. However, the $n=2$ states remain unstable until a (subcritical) steady state bifurcation at $\mathrm{Ra}^{\prime} \approx 1935$ gives rise to a mixed mode. Thus there are no stable steady states in the interval $1826<\mathrm{Ra}^{\prime}<1935$. Since the TW themselves lose stability at a tertiary Hopf bifurcation producing modulated traveling waves $(\mathrm{MW})$ at $\mathrm{Ra}^{\prime} \approx 1856$ the dynamics in $1856<\mathrm{Ra}^{\prime}<1935$ must have nontrivial time-dependence. In the remainder of this paper we describe some of the solutions we have located in this interval using direct numerical simulation of Eqs. (2a)-(2e). The integration in time is carried out using a semi-implicit second-order stiffly stable scheme, Fourier-Galerkin in $x$ and Chebyshev collocation in the $z$ direction. The Fourier nonlinear term is calculated pseudospectrally, increasing the number of modes to obtain a de-aliased evaluation. A minimum of 32 vertical points and 24 Fourier modes is used.

The loss of stability of the TW so soon after they bifurcate from the $n=1$ branch is discussed by Prat et al. [2002], and is simple to understand in the context of unfolding of the codimension-two bifurcation that arises when the parity-breaking bifurcation to the TW coincides with the saddle-node bifurcation on the $n=1$ branch. This bifurcation is a pitchfork-saddle-node bifurcation, and contains a secondary Hopf bifurcation in its unfolding that corresponds to the bifurcation of the TW to MW. This bifurcation moves farther up the TW branch as the codimension-two bifurcation is split apart, but continues to destabilize the TW at larger amplitude. Figure 4 shows the change in the MW as $\mathrm{Ra}^{\prime}$ increases beyond $\mathrm{Ra}^{\prime}=1856$ in terms of $\operatorname{Re} \theta_{2}(z, t)$ at the dimensionless location $z=-0.28$, where $\theta_{2}(z, t)$ is the $n=2$ Fourier amplitude of the temperature $\theta(x, z, t)$. The torus bifurcation at $\mathrm{Ra}^{\prime}=1856$ introduces a new frequency $\omega$ into the dynamics. Although $\omega \approx 4 \omega_{1}=2 \omega_{2}$ at the torus bifurcation the new frequency is incommensurate with $\omega_{j}$, and the resulting motion is quasiperiodic; the modulation frequency $\omega-2 \omega_{2}$ is clearly visible in Fig. 4. Here $\omega_{j}$ is the frequency of $\theta_{j}$, and in a traveling wave $\omega_{j}=j \omega_{1}, j \geq 1$. Since the frequency $\omega_{2}$ corresponds to drift it is absent from the mean temperature gradient which oscillates with the frequency $\omega$. The period of the Nusselt number is therefore $2 \pi / \omega$, and since $\omega \approx 2 \omega_{2}$, this period is approximately half the period of the fast oscillations in Fig. 4. These results should be compared with the corresponding ones for a problem with Boussinesq symmetry [Deane et al., 1987].

Figure 4 shows that the modulation period increases dramatically near $\mathrm{Ra}^{\prime}=1863$, suggesting the presence of a global bifurcation. Figure 5 confirms this impression and compares the modulation period with the Nusselt number period as functions 


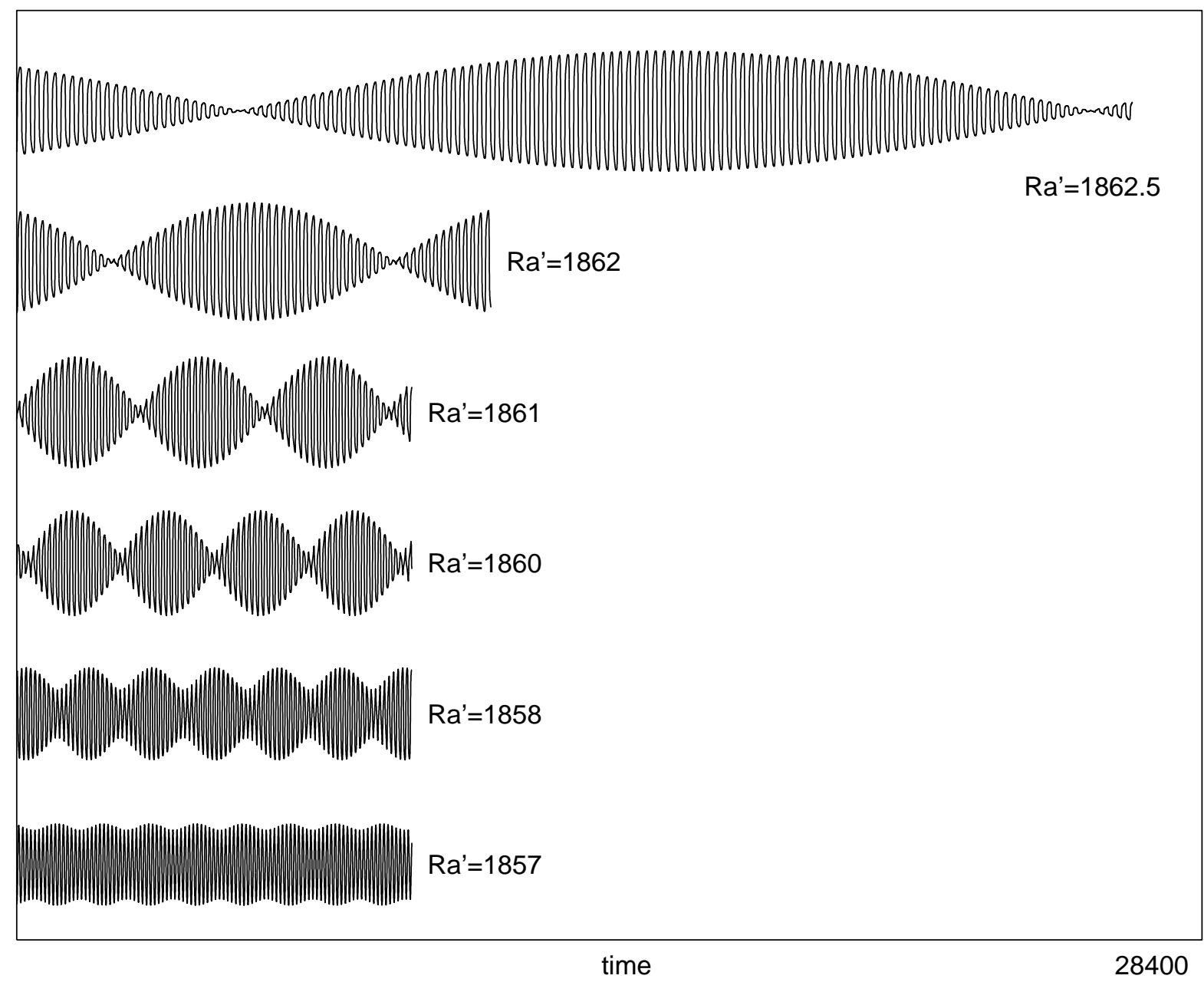

Fig. 4. Time series $\operatorname{Re} \theta_{2}(z=-0.28, t)$ for several values of $\mathrm{Ra}^{\prime}$ near the global bifurcation at $\mathrm{Ra}^{\prime} \approx 1863$.

(a)

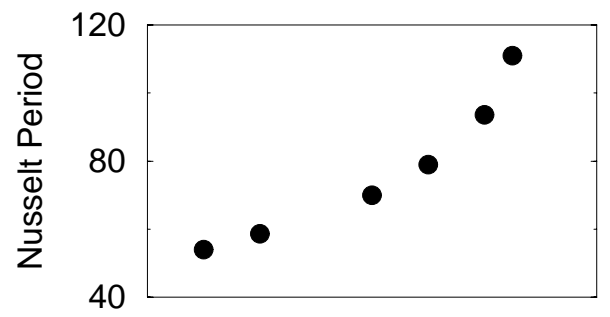

(b)

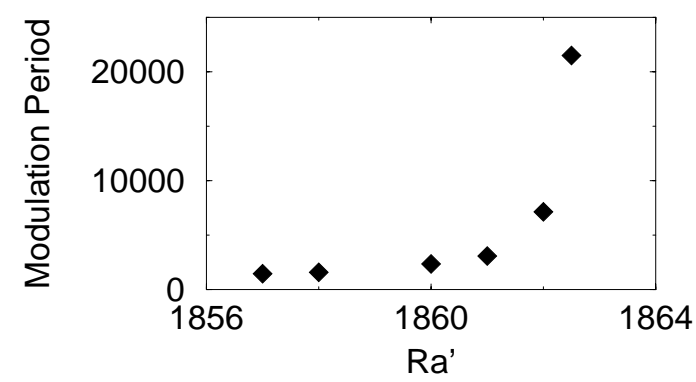

Fig. 5. (a) The Nusselt number period and (b) the modulation period from Fig. 4 as functions of $\mathrm{Ra}^{\prime}$. Both diverge at $\mathrm{Ra}^{\prime} \approx 1863$. of $\mathrm{Ra}^{\prime}$ in this regime. Near the global bifurcation the period $2 \pi / \omega_{2}$ of the $\theta_{2}$ oscillation is double that in the Nusselt number and both diverge together. The simulations indicate that this divergence is a consequence of the approach of the phase space trajectory closer and closer to reflection-invariant subspaces. As this occurs the Eulerian mean flow $U$ associated with the MW disappears (Fig. 6). This flow is periodic when the wave is a modulated traveling wave, and oscillates with the same period as the Nusselt number. Figure 7 shows a projection of these solutions onto the complex $\theta_{1}$ plane. At $\mathrm{Ra}^{\prime}=1857$ the modulation is weak and the oscillation is largely azimuthal, with the trajectory circumscribing the origin in a counterclockwise direction. The corresponding state in physical space is a left-traveling wave with a small amplitude modulation superposed on it. By the time $\mathrm{Ra}^{\prime}=1862.5$ the oscillations are primarily radial indicating that the 


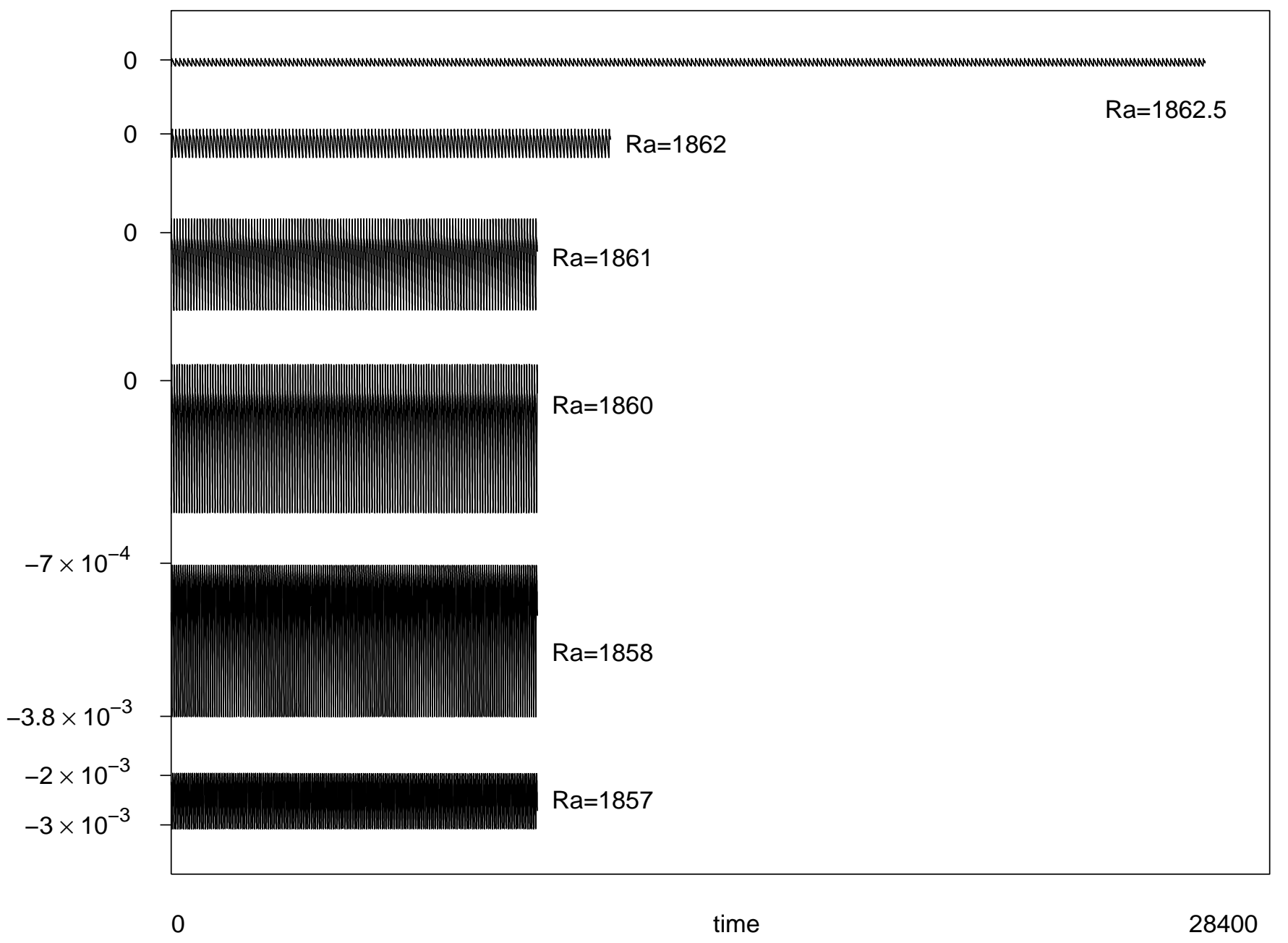

Fig. 6. The mean flow $U(z, t)$ versus time at $z=-0.28$ for several different values of $\mathrm{Ra}^{\prime}$ near the global bifurcation at $\mathrm{Ra}^{\prime} \approx 1863$ showing the rapid disappearance of the mean flow as the modulated traveling wave approaches the circle of reflection-invariant subspaces.

amplitude modulation dominates the slow residual drift in the counterclockwise direction that remains. The frequency $\omega_{2}$ is now associated with the standing component of the oscillation, and the Nusselt number frequency is therefore $2 \omega_{2}$, as observed; the drift frequency corresponds to the modulation frequency shown in Fig. 5(b).

A global bifurcation occurs when the modulation period diverges [cf. Fig. 5(b)], i.e. when the MW collides with the circle of $n=2$ fixed points. Each of these fixed points may be labeled by its spatial phase $\phi$, and lies in a reflection-invariant subspace we call $\Sigma_{\phi}$. In the situation considered here the subspaces $\Sigma_{\phi}$ contain no standing waves, and consequently the Nusselt number period must diverge at the same value of $\mathrm{Ra}^{\prime}$ as the modulation period. At the global bifurcation a particular MW trajectory approaches one of the $n=2$ fixed points, in $\Sigma_{\phi}$ say, and thereafter remains trapped in $\Sigma_{\phi}$. Its evolution in $\Sigma_{\phi}$ corresponds to a reorientation (over infinite time) of the trajectory relative to the $n=1$ state, followed by a transition (also over infinite time) to an $n=2$ state with the opposite sign. During the first phase the amplitude of the $n=1$ component remains zero; during the second phase the $n=1$ mode is briefly excited but the spatial phase of both modes remains fixed. The resulting heteroclinic cycle thus consists of the destruction of an $n=2$ state followed by its reconstruction with the opposite sign. This new state can be thought of as an $L / 4$ translate of the original $n=2$ state. Within the normal form for the $1: 2$ spatial resonance this global bifurcation transfers stability from the MTW to a structurally stable heteroclinic cycle connecting $n=2$ steady states with their translates by $L / 4$ (see Sec. 4). Such cycles can 

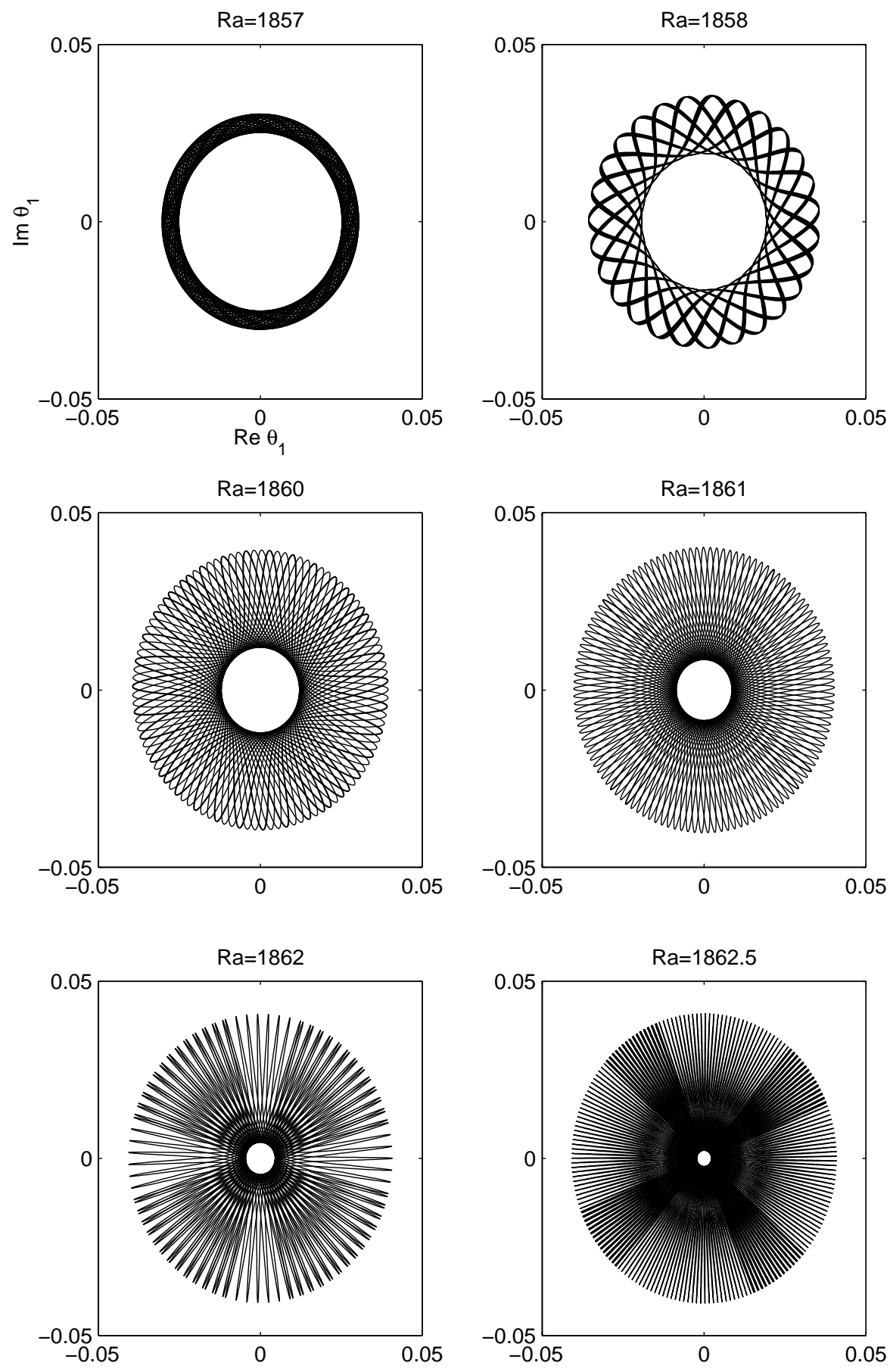

Fig. 7. Projections of the solutions in Figs. 4 and 6 on the complex $\theta_{1}(z=-0.28)$ plane, showing that the solutions correspond to quasiperiodic MW. The gradual transition from (counterclockwise) azimuthal to radial oscillation is a consequence of the approach of the (left-traveling) MW to a circle of reflection-invariant subspaces. Near $\mathrm{Ra}^{\prime}=1863$ the MW travels only during the brief transitions from the vicinity of one invariant subspace to the next.

therefore be thought of as heteroclinic waves of the type just described, but ones that spend arbitrarily long intervals of time in the stationary state. We may expect something similar to occur in the pdes (2a) $-(2 \mathrm{e})$ as well, even though there is no definitive proof that such a global bifurcation in fact takes place. However, assuming that it does we may use the theory of Krupa and Melbourne [1995] to confirm that the resulting heteroclinic cycle is asymptotically stable. Its stability is determined by the leading eigenvalues of $n=2$ state. This state has one zero eigenvalue generated by translations and 


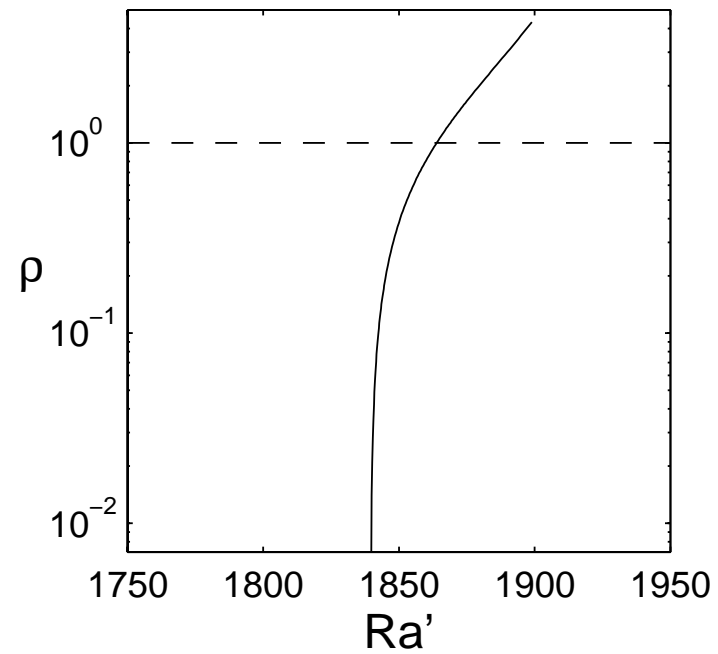

Fig. 8. The eigenvalue ratio $\rho$ (see text) as a function of $\mathrm{Ra}^{\prime} ; \rho>1$ indicates that the (putative) structurally stable heteroclinic cycle is attracting.

one unstable eigenvalue in the reflection-invariant subspace (corresponding to $n=1$ perturbations) which we call $\lambda^{+}$. All the remaining eigenvalues are stable, and we divide them into two groups, those with eigenvectors in the reflection-invariant subspace, and those perpendicular to it. We denote the (absolute value of the real part of the) least stable perpendicular eigenvalue by $\lambda^{-}$; this is the eigenvalue whose eigenvector defines the direction along which the MW trajectory approaches the $n=2$ fixed points. Under these conditions the theorem of Krupa and Melbourne [1995] implies that the heteroclinic cycle connecting two $n=2$ fixed points related by an $L / 4$ translation is attracting provided $\rho \equiv \lambda^{-} / \lambda^{+}>1$. Note that the eigenvector of $\lambda^{+}$ for an $n=2$ state and that of $\lambda^{-}$for its $L / 4$ translate are both reflection-invariant with respect to the same reflection. Figure 8 shows $\rho$ as a function of $\mathrm{Ra}^{\prime}$, and confirms that the cycle acquires stability for $\mathrm{Ra}^{\prime} \gtrsim 1863$, i.e. beyond the termination of the MW branch identified in Fig. 5(b). However, the resulting theory predicts that in $\mathrm{Ra}^{\prime} \gtrsim 1863$ the excursions between the two fixed points should occur more and more infrequently as time progresses, and the trajectory comes closer and closer to the heteroclinic cycle. In fact in our simulations we find that the oscillation period saturates (Fig. 9), and

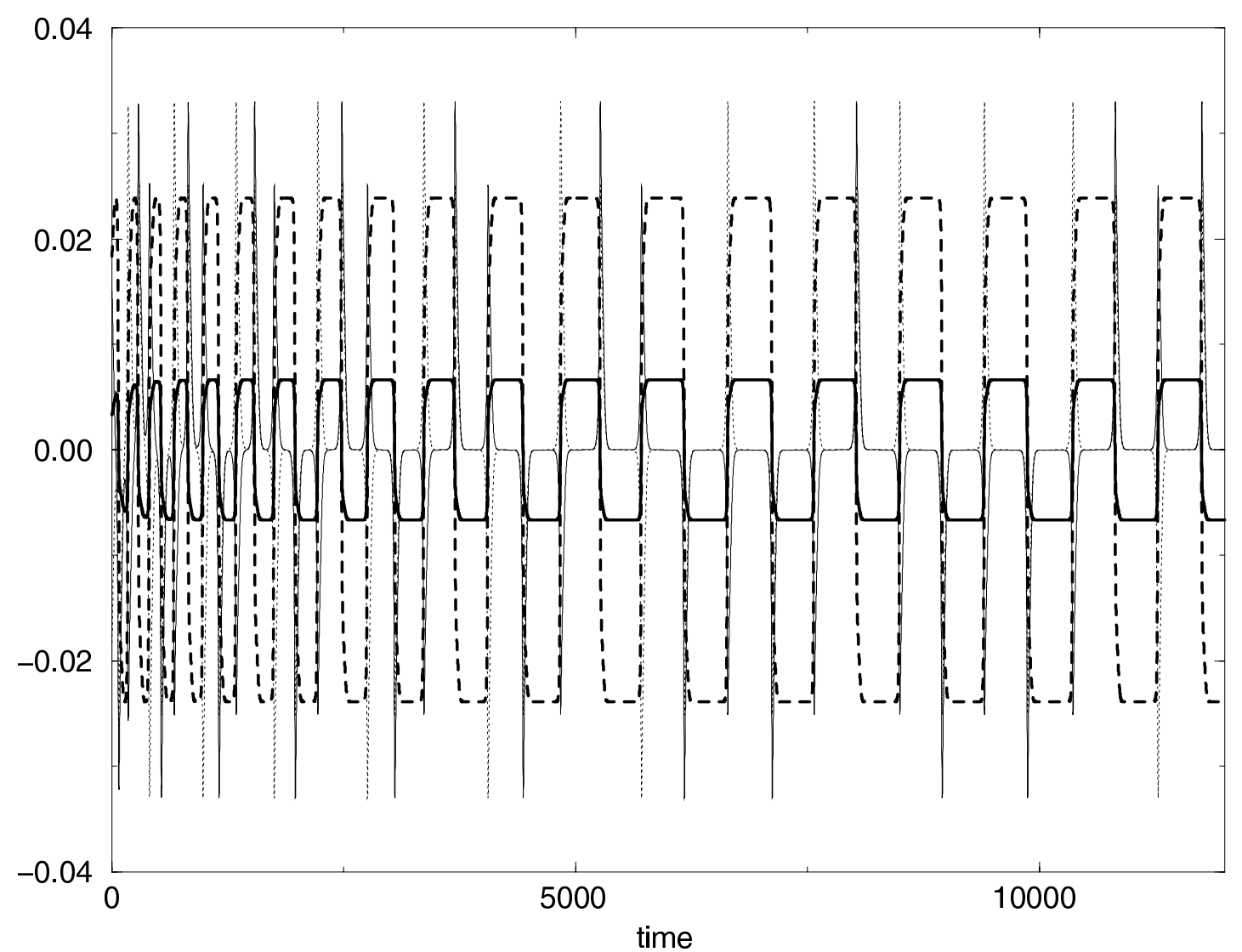

Fig. 9. Time series $\operatorname{Re} \theta_{1}(-), \operatorname{Im} \theta_{1}(--)$, and $\operatorname{Re} \theta_{2}(-), \operatorname{Im} \theta_{2}(---)$ (heavy lines) at $z=-0.28$ for $\operatorname{Ra}^{\prime}=1865$ showing the gradual lengthening of the oscillation period, followed by saturation. 
we (may) obtain a periodic cycle with finite albeit long period [Fig. 10(a)]. In this plot (as in Fig. 9) the heavy line shows $\operatorname{Re} \theta_{2}(z=-0.28, t)$; we see that the system spends extended periods of time in an almost pure $n=2$ state. The thin lines in turn show the real (solid) and imaginary (dashed) parts of $\theta_{1}(z=-0.28, t)$; these have twice the period of $\theta_{2}(z=-0.28, t)$, as expected of a TW, and are only excited during the brief transitions from one $n=2$ state to its $L / 4$ translate. In the example shown the phase of $\theta_{1}$ rotates clockwise, corresponding to a pattern that translates, in fits and starts, towards the right. Integration at $\mathrm{Ra}^{\prime}=1870$ with a time step $\Delta t=4 \times 10^{-3}$ shows that the saturated period in $\theta_{1}$ fluctuates from period to period by at most $0.2 \%$; this period changes by $0.4 \%$ when the time step is decreased by a factor of 10 . Similar results apply to $\mathrm{Ra}^{\prime}=1865$. In contrast, Fig. 10(b) shows a time series, also for $\mathrm{Ra}^{\prime}=1865$, that appears to remain chaotic for all time, albeit it still with a well-defined switching time. The two time series differ in the initial conditions employed, and identical time series were obtained with a different code using primitive variables [Karniadakis et al., 1991], provided both were started from the same initial conditions. Specifically, if both codes are started from the same small disturbance in $\operatorname{Re} \theta_{1}$ they produce identical periodic time series. Moreover, when the same time step is used the periods calculated with the two codes differ by only $0.6 \%$. In contrast, if the calculation is started from a general (complex) perturbation in $\theta_{1}$ the time series remains irregular (chaotic) for as long as the integration is carried out (cf. Zhou and Sirovich [1992]), but once again both codes yield identical time series. These results suggest that the periodicity (or the chaotic behavior) of the time series is not an artifact of numerical discretization or round-off errors, even though we do detect a very slow drift in $\operatorname{Im} \theta_{2}$ with both methods (which we attribute to the presence of a zero eigenvalue arising from translation invariance of the system).

The characteristic time $\tau$ between successive switches in $\theta_{1}$ also depends on initial conditions. However, the overall oscillation amplitude is the same in both cases, i.e. the fixed points visited by the cycle are identical. The amplitudes in Figs. 10(a) and 10(b) look different only because the imaginary part of $\theta_{2}$ vanishes in Fig. 10(a) but is nonzero in Fig. 10(b). Similar results obtain for $\mathrm{Ra}^{\prime}=1870$ and $\mathrm{Ra}^{\prime}=1885$, albeit with larger

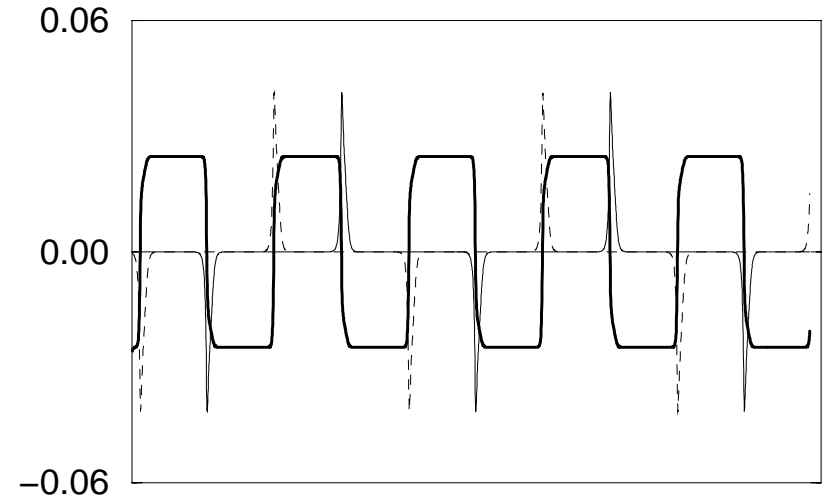

(a)

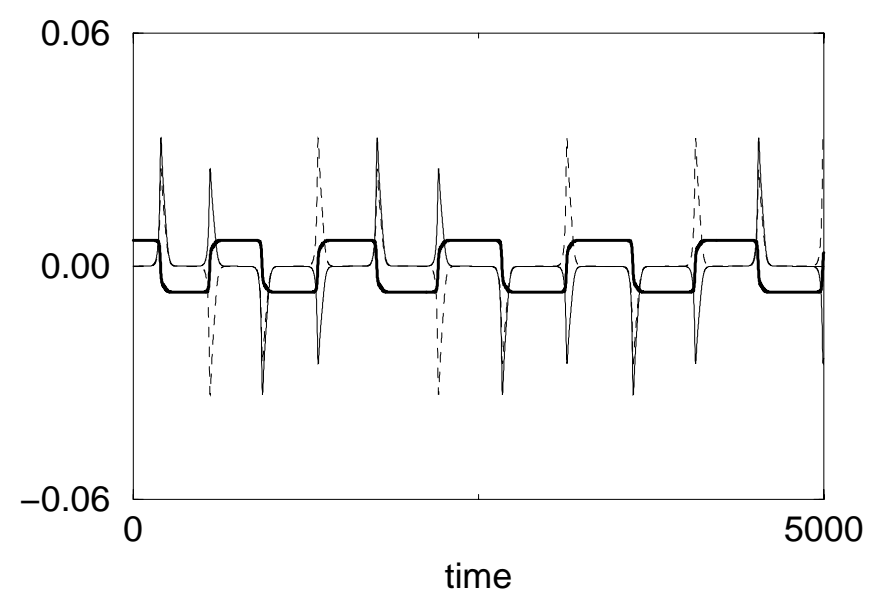

(b)

Fig. 10. Long time behavior of $\operatorname{Re} \theta_{1}(-), \operatorname{Im} \theta_{1}(--)$, and $\operatorname{Re} \theta_{2}$ (heavy line) at $z=-0.28$ for two different initial conditions when $\mathrm{Ra}^{\prime}=1865$. In (a) the cycle is periodic with $\tau \approx 490$; in (b) it is irregular with $\tau \sim 460$.

values of $\tau$. In the periodic case the asymptotic pe$\operatorname{riod} T$ of the time series is $4 \tau$, with the periods of $\theta_{j}, j=1,2$, given by $T_{j}=4 \tau / j$. In the chaotic case $\theta_{2}$ remains approximately periodic, with $T_{2} \approx 2 \tau$.

In an attempt to shed light on the initial condition dependence of the results we computed the quantity $\theta \equiv \arg \theta_{2}-2 \arg \theta_{1}$ as a function of time. This quantity is invariant under translations (cf. Sec. 4). If $\theta=0(\bmod \pi)$ the pattern does not drift and the dynamics then takes place in an invariant subspace. We find that in all cases $\theta=0$ almost all the time, although during each cycle $\dot{\theta}$ becomes briefly nonzero as the phase of the $n=1$ component reorients itself prior to the switch from the $n=2$ state to its $L / 4$ translate. During the rest of the cycle $\theta$ is essentially zero, and it is during this phase of the oscillation that the switch between the two $n=$ 2 states takes place. Since this switch corresponds 


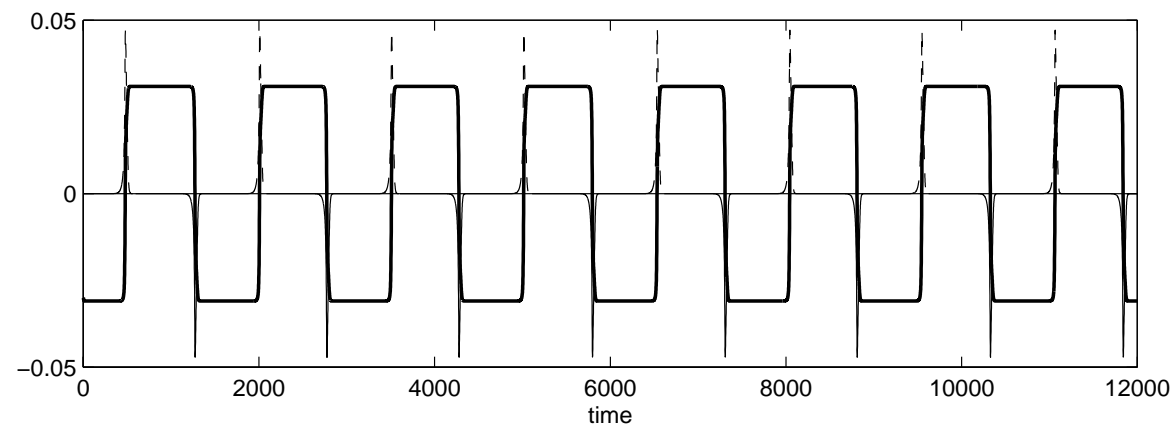

(a)

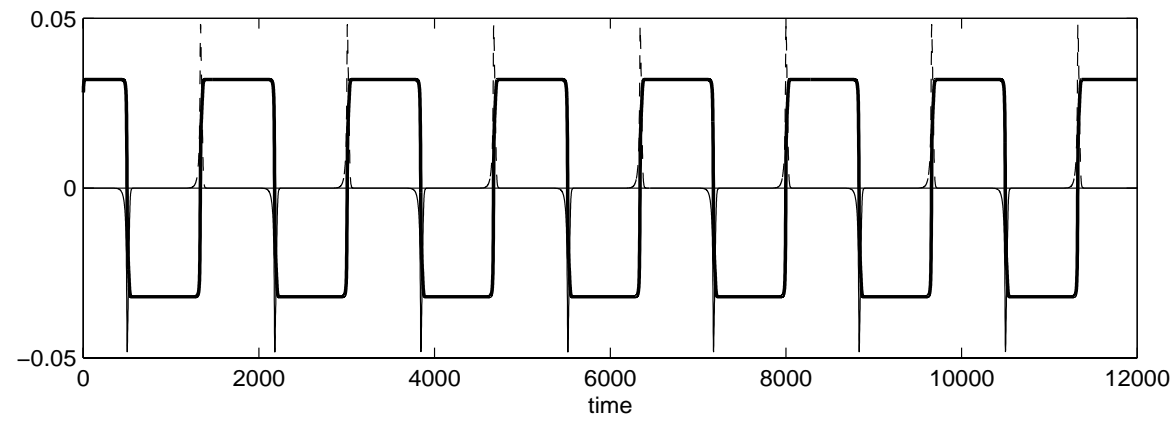

(b)

Fig. 11. Time series $\operatorname{Re} \theta_{1}(-), \operatorname{Im} \theta_{1}(---)$, and $\operatorname{Re} \theta_{2}$ (heavy line) at $z=-0.28$ after extended transients for (a) $\operatorname{Ra}^{\prime}=1890$,

(b) $\mathrm{Ra}^{\prime}=1895$. Note that in contrast to Fig. 10 all three variables ultimately oscillate with the same period $T$, with (a) $T \approx 1500$, (b) $T \approx 1660$.

to a $\pi$ jump in $\arg \theta_{2}$ it is accompanied by a $\pi / 2$ jump $\arg \theta_{1}$ as seen in Figs. 10(a) and 10(b). Thesejumps are all clockwise in the initial segment of the time series in Fig. $9(t<7100)$ but become disordered following the first counterclockwise jump at $t \approx 7100$. These results indicate that the dynamics do not take place in an invariant subspace, although they are very close to being in one. As discussed in Sec. 4 these facts are consistent with the normal form theory for this problem, and indicate that the initial conditions do not confine the dynamics to an invariant subspace.

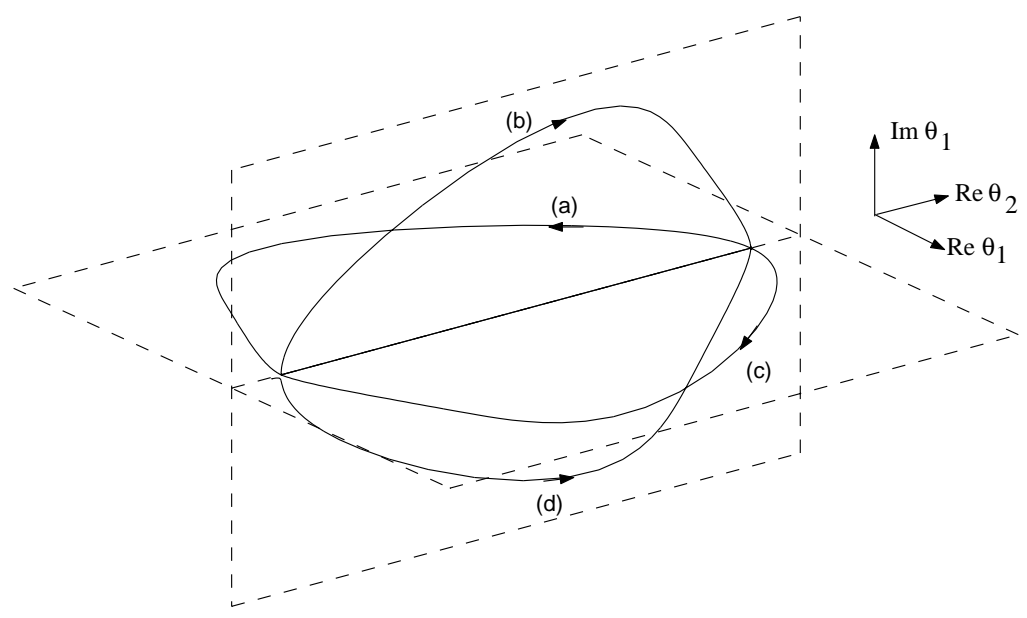

Fig. 12. The four (nearly) heteroclinic connections, labeled (a-d), between an $n=2$ state and its $L / 4$ translate at $\mathrm{Ra}^{\prime}=1865$ projected onto the three-dimensional space $\left(\operatorname{Re} \theta_{1}, \operatorname{Im} \theta_{1}, \operatorname{Re} \theta_{2}\right)$. 
Figures 11(a) and 11(b) show similar time series for $\mathrm{Ra}^{\prime}=1890$ and $\mathrm{Ra}^{\prime}=1895$, in both cases starting from a periodic solution at $\mathrm{Ra}^{\prime}=1885$. Careful integration of the equations shows that after a very long transient the system again settles into a periodic switching sequence, but this time with $\operatorname{Re} \theta_{1}, \operatorname{Im} \theta_{1}$ and $\operatorname{Re} \theta_{2}$ having identical periods, i.e. $T_{1}=T_{2}=T / 2$. It follows that for these values of $\mathrm{Ra}^{\prime}$ the sequence of transitions no longer follows the dynamics of the modulated traveling wave $t=200$

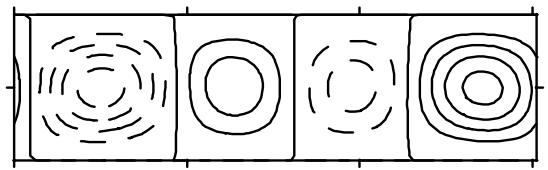

$t=180$

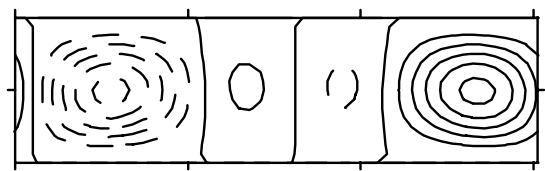

$t=160$

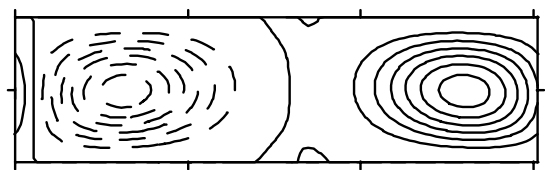

$t=140$

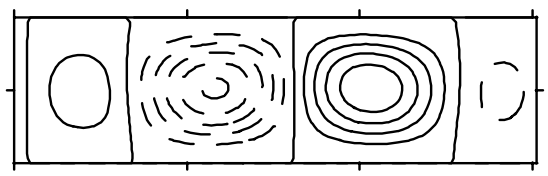

$t=120$

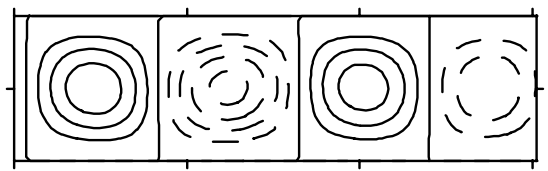

$t=100$

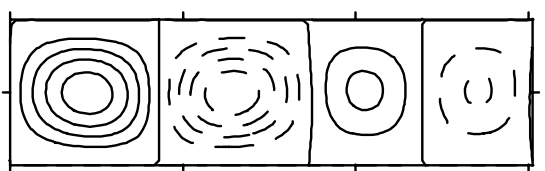

$t=80$

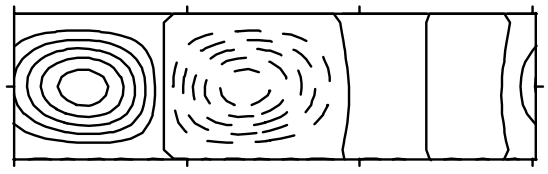

$t=60$

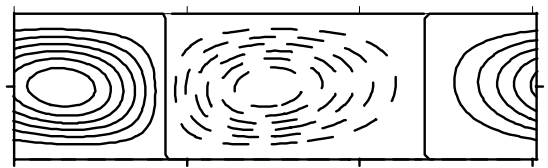

$t=40$

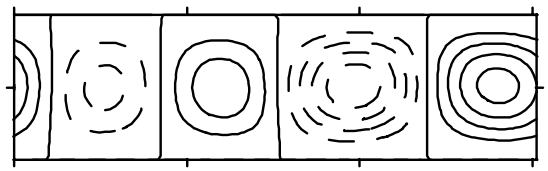

$t=20$

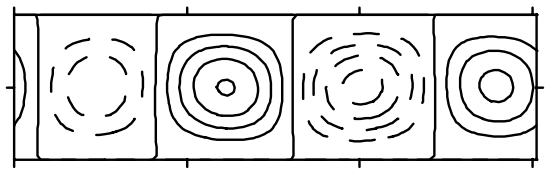

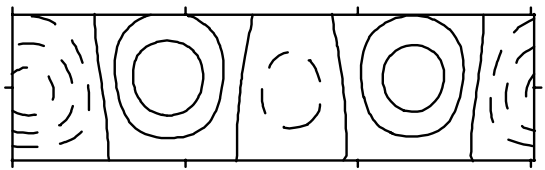
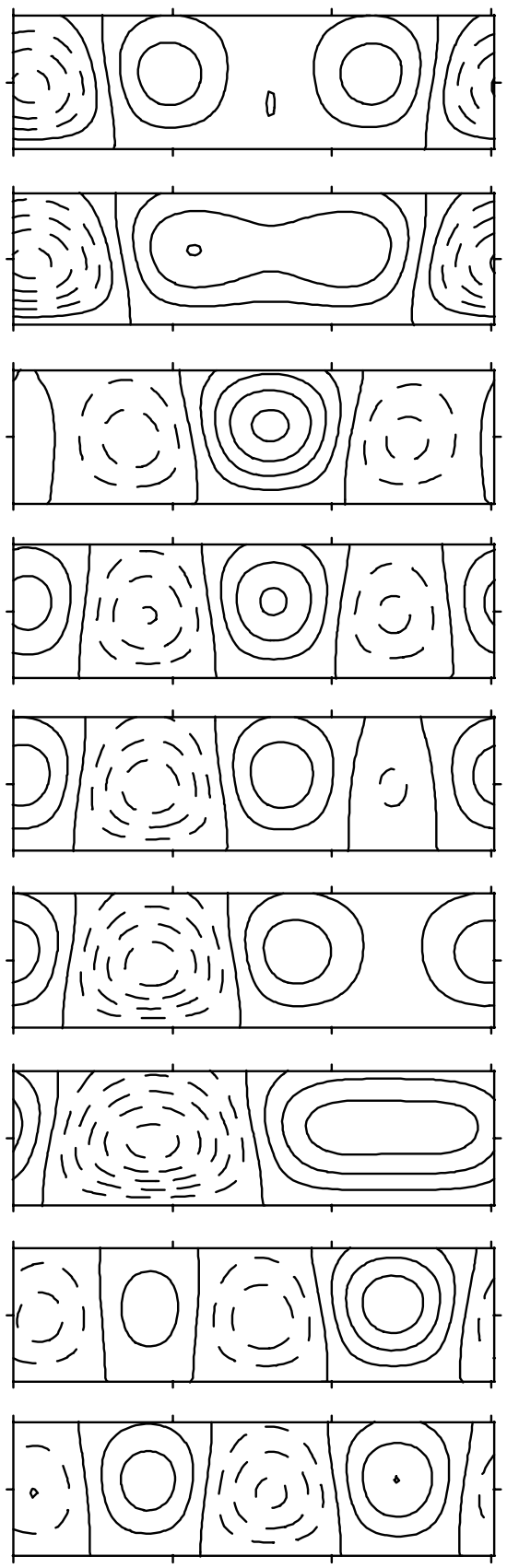

Fig. 13. A series of snapshots of the instantaneous streamlines of $(U, 0)+\left(-\partial_{z} \chi^{\prime}, \partial_{x} \chi^{\prime}\right)$ (left panels) and the temperature fluctuation $\theta$ (right panels) at $\mathrm{Ra}^{\prime}=1862$, with time increasing upwards. The pattern drifts towards the left in fits and starts, with a translation by $L / 4$ occurring in time $T / 4 \approx 100$. 
prior to the acquisition of stability by the structurally stable heteroclinic cycle. Instead the phase of $\theta_{1}$ oscillates back and forth. We may visualize the resulting cycle in terms of Fig. 12. This figure shows the heteroclinic connections in the threedimensional space $\left(\operatorname{Re} \theta_{1}, \operatorname{Im} \theta_{1}, \operatorname{Re} \theta_{2}\right)$. In a rotating wave [Fig. 10(a)] the system switches from connection (a) to (b) to (c) and then to (d), or the reverse. In the time series in Figs. 11(a) and 11(b) the system switches from (b) to (a) and then back to (b). We can also find time series corresponding to repeated switches between (b) and (c), and surmise that at least four stable long period periodic orbits are present for these values of $\mathrm{Ra}^{\prime}$. The nature of the transition from rotating phase to trapped phase with increasing $\mathrm{Ra}^{\prime}$ is not understood, but it is not the result of a change in the order of the leading eigenvalues of $n=2$ state. However, the location of the transition appears to be well-defined: if one starts with a settled solution at $\mathrm{Ra}^{\prime}=1890$ with trapped phase and reduces $\mathrm{Ra}^{\prime}$ to $\mathrm{Ra}^{\prime}=1885$ the time series ultimately develops rotating wave symmetry.

Figure 13 gives a good idea of what is happening in the physical system in an MW state close to the global bifurcation at $\mathrm{Ra}^{\prime} \approx 1863$. The figure shows the instantaneous streamlines (left panels) and $\theta$ (right panels) at $\mathrm{Ra}^{\prime}=1862$ with time increasing upwards, and reveals the gradual translation of the pattern towards the left (corresponding to counterclockwise rotation in Fig. 7; see Fig. 14 for the corresponding time series). The patterns spend dominant periods of time in a state characterized by wavenumber $n=2$ but undergo welldefined episodes during which the $n=1$ mode is excited, leading to the disruption of the pattern followed by its regrowth as an $L / 4$ translate. In the figure these episodes manifest themselves by a temporary doubling of the wavelength of the pattern; these larger cells then undergo "fission" due to the regrowth of a counterrotating cell in the center of these larger cells. This type of dynamic is well-known from studies of the breakdown of the turbulent boundary layer [Aubry et al., 1988; Holmes et al., 1996]. Figure 14 shows the associated (periodic) oscillations in the Nusselt number, the mean flow and the conventionally defined Rayleigh number Ra; these oscillations reflect the oscillations in the amplitude of the wave. The dynamic associated with the nearly heteroclinic cycles discussed above is almost the same, except that the time spent near the pure $n=2$ states is longer. Figure 15
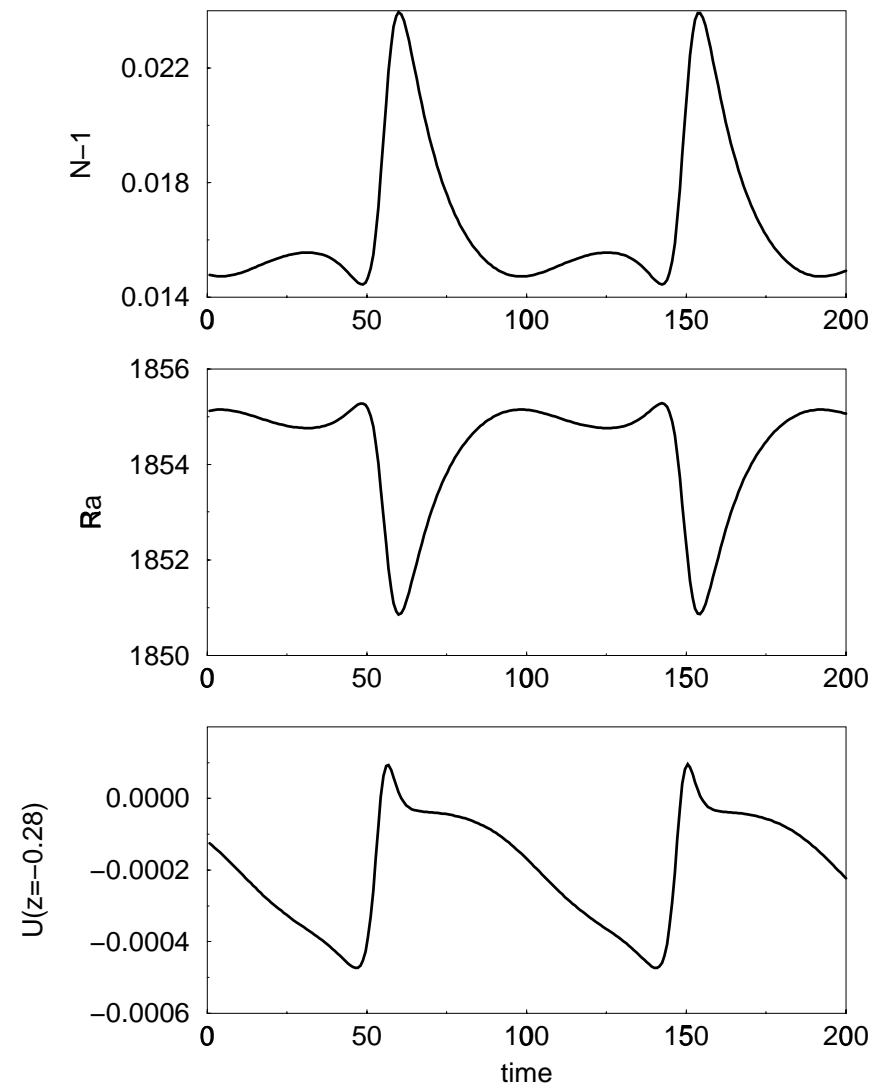

Fig. 14. The time series for $N-1$, Ra and $U(z=-0.28)$ for a modulated traveling wave when $\mathrm{Ra}^{\prime}=1862$, showing that the conventionally defined Rayleigh number (1c) fluctuates for states with a nonzero amplitude modulation whenever $B^{*} \neq 1$ at either top or bottom (or both).

shows the details of the four transitions that together make up the cycle in Fig. 10(a).

\section{2. $B_{+}^{*}=1, B_{-}^{*}=0$}

For these values of the Biot number the 1:2 mode interaction takes place at $\mathrm{Ra}^{\prime}=1463.19, k=1.77$. The results reported in Fig. 16 are for $\sigma=0.1$, $k=1.75$. Since $B_{-}^{*}=0$ (so that $N=1$ for both steady states and traveling waves) the figure shows instead $\left(\mathrm{Ra}^{\prime}-\mathrm{Ra}\right) / \mathrm{Ra}$ versus $\mathrm{Ra}^{\prime}$. For this choice of $k$ the first instability is to an $n=2$ mode, at $\mathrm{Ra}^{\prime}=1448.01$, and is supercritical; this branch remains stable until $\mathrm{Ra}^{\prime}=1455$, where stability is transferred to the $n=1$ branch. The latter originates in a subcritical bifurcation at $\mathrm{Ra}^{\prime}=1476.38$ and undergoes two successive bifurcations before its termination on the $n=2$ branch: the first, at $\mathrm{Ra}^{\prime}=1467.2$, is a parity-breaking bifurcation and produces a branch of (unstable) traveling waves; the 
second is a supercritical Hopf bifurcation that stabilizes the $n=1$ branch and produces a branch of stable standing waves (SW). The resulting bifurcation diagram (Fig. 16) resembles Figs. 14(i) and $14(\mathrm{j})$ of Prat et al. [2002]. In Fig. 17 we show the standing waves in the $\left(\operatorname{Re} \theta_{1}, \operatorname{Re} \theta_{2}\right)$ plane for several values of $\mathrm{Ra}^{\prime}$; for these solutions $\operatorname{Im} \theta_{1}$ and $\operatorname{Im} \theta_{2}$ both vanish. As $\mathrm{Ra}^{\prime}$ increases the period of the standing waves increases rapidly and a hetero- clinic connection between the origin and the $n=2$ fixed point forms at $\mathrm{Ra}^{\prime} \approx 1461.3$. As before, such connections form in every reflection-invariant subspace. For larger $\mathrm{Ra}^{\prime}$ we find that the system follows a nearly heteroclinic cycle, of the same type as shown in Fig. 10(a). Figure 18 shows an example for $\mathrm{Ra}^{\prime}=1461.4$. Once again the trajectory appears to be periodic in time, albeit with a long period, in contrast to theory which predicts
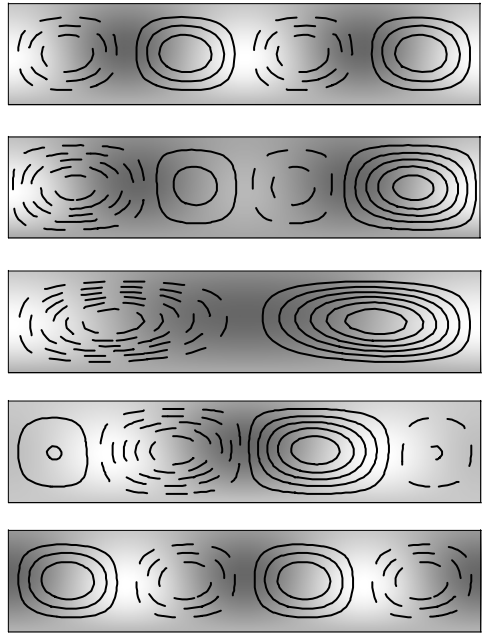

(a)
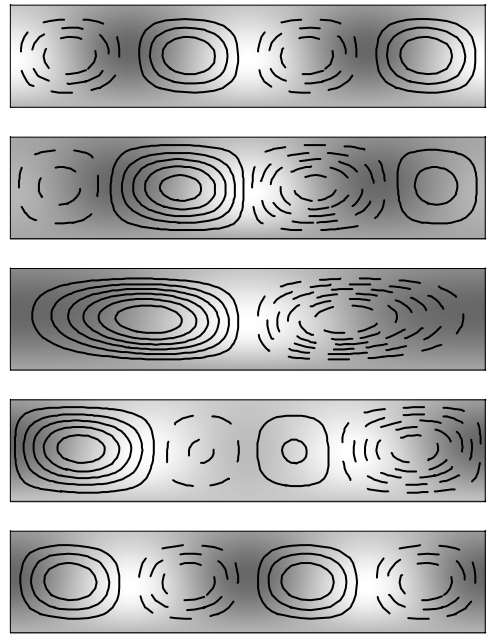

(c)
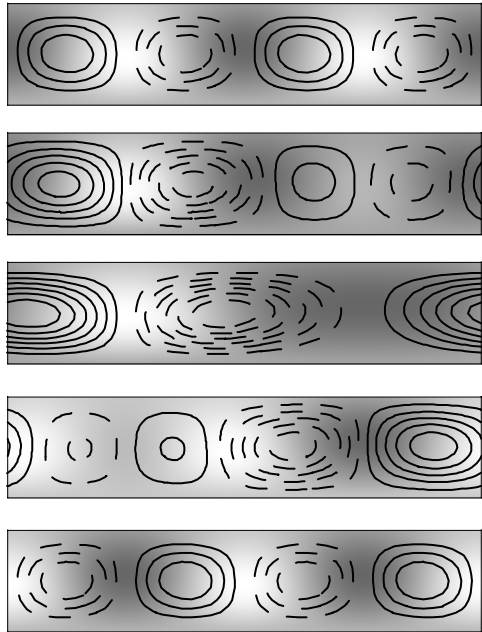

(b)
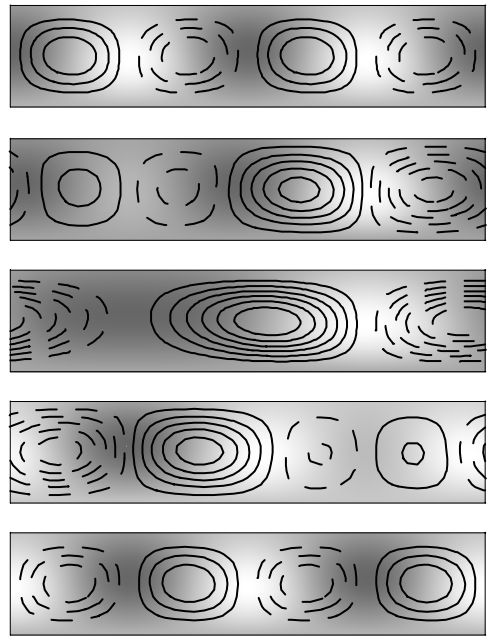

(d)

Fig. 15. The details of the four excursions that form the (near-) heteroclinic cycle at $\mathrm{Ra}^{\prime}=1865$ [Fig. 10(a)], in terms of the instantaneous streamlines superposed on the temperature fluctuations (shaded), with time increasing upwards. (a) The transition from $\operatorname{Re} \theta_{2}>0$ to $\operatorname{Re} \theta_{2}<0$ via $\operatorname{Re} \theta_{1}>0$, (b) the transition from $\operatorname{Re} \theta_{2}<0$ to $\operatorname{Re} \theta_{2}>0$ via $\operatorname{Im} \theta_{1}<0$, (c) from $\operatorname{Re} \theta_{2}>0$ to $\operatorname{Re} \theta_{2}<0$ via $\operatorname{Re} \theta_{1}<0$, and (d) from $\operatorname{Re} \theta_{2}<0$ to $\operatorname{Re} \theta_{2}>0$ via $\operatorname{Im} \theta_{1}>0$. 


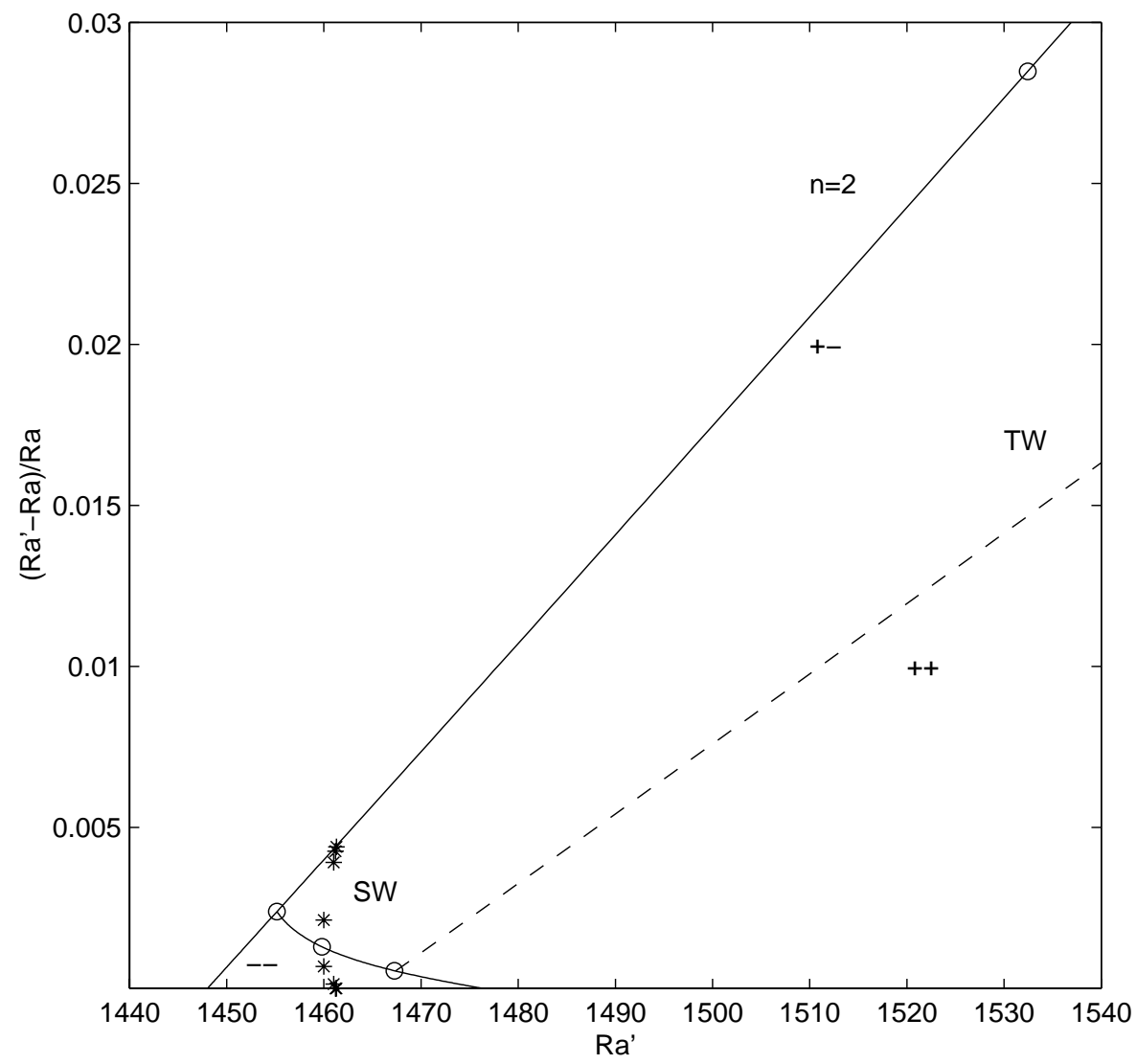

Fig. 16. Bifurcation diagram $\left(\mathrm{Ra}^{\prime}-\mathrm{Ra}\right) / \mathrm{Ra}$ versus $\mathrm{Ra}^{\prime}$ for $\sigma=0.1, B_{+}^{*}=1, B_{-}^{*}=0$ and $k=1.75$, showing steady states $(-)$, TW (-- ) and SW (*); open circles denote local bifurcations. For the $\mathrm{SW}$ both $\left(\mathrm{Ra}^{\prime}-\mathrm{Ra}_{\max }\right) / \mathrm{Ra}_{\max }$ and $\left(\mathrm{Ra}^{\prime}-\mathrm{Ra}_{\min }\right) / \mathrm{Ra}_{\mathrm{min}}$ are plotted. A bifurcation to stable standing waves occurs on the $n=1$ branch.

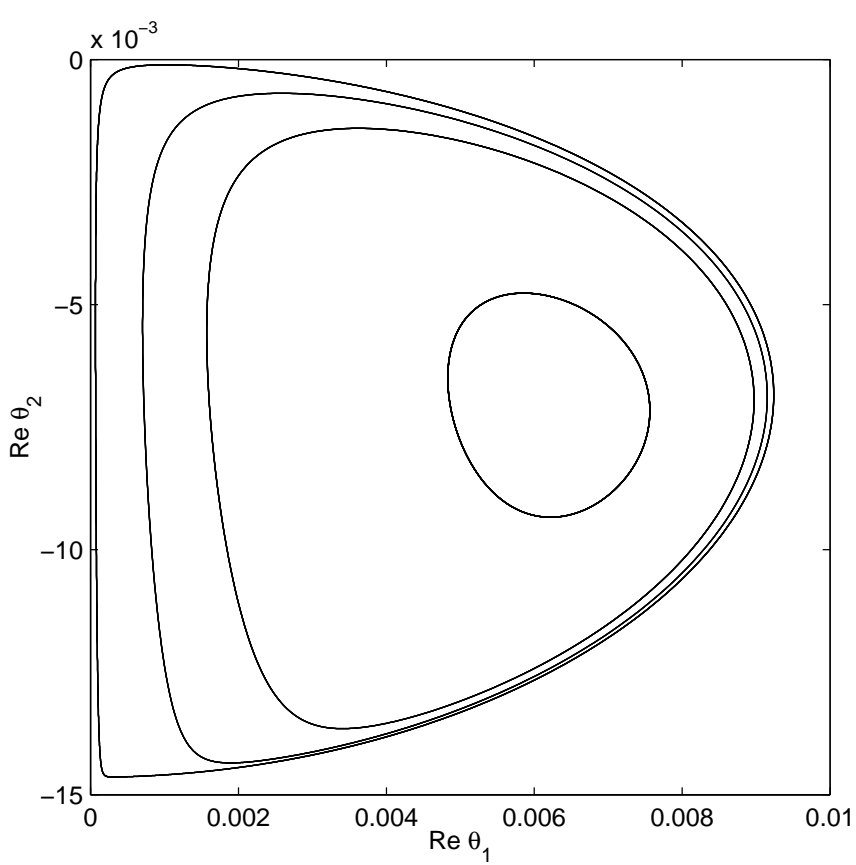

Fig. 17. The stable standing waves projected onto the $\left(\operatorname{Re} \theta_{1}, \operatorname{Re} \theta_{2}\right)$ plane at $z=-0.28$ for $\mathrm{Ra}^{\prime}=1460,1461$, 1461.2 and 1461.3, showing the approach to a heteroclinic connection between the origin and the $n=2$ fixed point. a gradual lengthening of the period as the trajectory approaches closer and closer to the putative heteroclinic cycle. Numerically we find that the characteristic interval between successive switches depends on the value of $\mathrm{Ra}^{\prime}$; we attribute this dependence to the dependence of $T$ on the eigenvalue ratio $\rho$. In the case under discussion the typical values of $T$ (see Fig. 11) are substantially larger than in Case (i), Sec. 3.1, a likely consequence of the substantially larger values of $\rho$.

\section{Discussion}

The results described in the preceding section bear a close resemblence to those familiar from studies of the normal form for the 1:2 spatial resonance with periodic boundary conditions on a line, given by the (scaled) equations [Dangelmayr, 1986; Armbruster et al., 1988; Proctor \& Jones; 1988; Porter \& Knobloch, 2001]

$$
\dot{z}_{1}=\left(\mu_{1}+d_{11}\left|z_{1}\right|^{2}+d_{12}\left|z_{2}\right|^{2}\right) z_{1}+\bar{z}_{1} z_{2}
$$


(a)

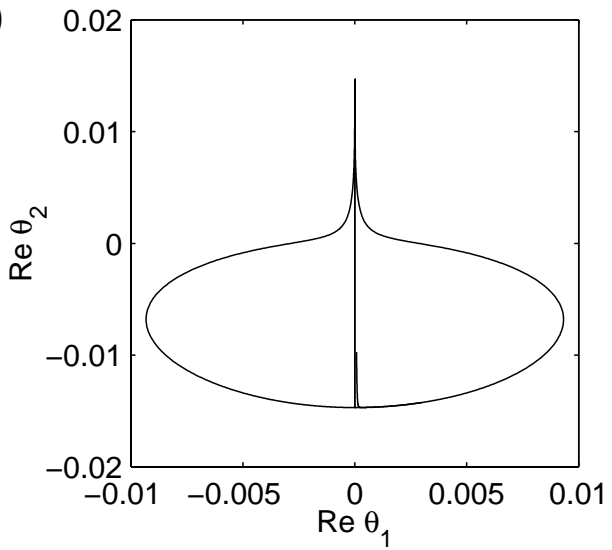

(b)

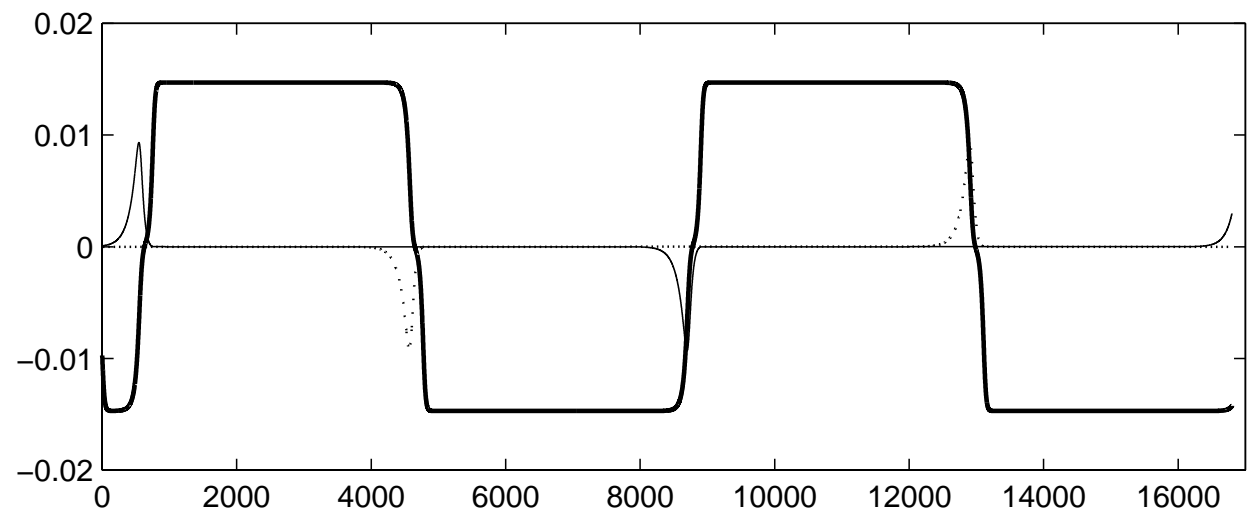

(c)

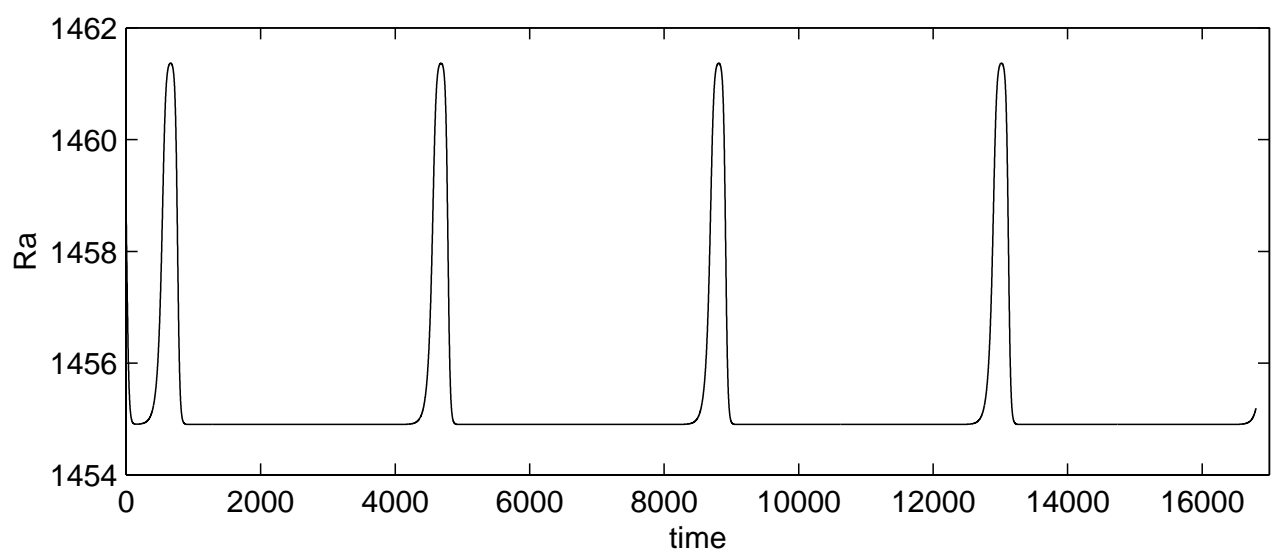

Fig. 18. A nearly heteroclinic trajectory at $\operatorname{Ra}^{\prime}=1461.4$. (a) Projection onto the $\left(\operatorname{Re} \theta_{1}, \operatorname{Re} \theta_{2}\right)$ plane at $z=-0.28$, (b) time series for $\operatorname{Re} \theta_{1}(-), \operatorname{Im} \theta_{1}(--)$, and $\operatorname{Re} \theta_{2}$ (heavy line) at $z=-0.28$, and (c) the corresponding oscillations in the Rayleigh number Ra defined in Eq. (1c).

$$
\dot{z}_{2}=\left(\mu_{2}+d_{21}\left|z_{1}\right|^{2}+d_{22}\left|z_{2}\right|^{2}\right) z_{2}+\sigma z_{1}^{2} .
$$

Here the $z_{j}$ are complex amplitudes of the eigenfunctions $\exp i j k x, j=1,2, \sigma= \pm 1$, and the equations have been truncated at third order. It should be noted that in systems of hydrodynamic type (i.e. systems with only advective nonlinearities) the coefficient $\sigma$ is necessarily -1 [Chossat, 2001]; this is the "interesting" case in which TW are found near the $1: 2$ resonance. Equations of the above type with $\sigma=-1$ have also been derived from the pdes describing two-dimensional weakly non-Boussinesq convection [Manogg \& Metzener, 1994]. From the point of view of bifurcation theory these equations apply rigorously only in situations in which the coefficients of both quadratic terms are small, resulting in a balance between the linear, quadratic and cubic terms. However, it is not necessary to think 
of this (near) degeneracy, and one may instead use these equations to examine the evolution of the dynamics described by Armbruster et al. [1988], and Proctor and Jones [1988] as one moves away from the mode interaction point, i.e. as $|\mu| \equiv\left(\mu_{1}^{2}+\mu_{2}^{2}\right)^{1 / 2}$ increases. It is in this sense that Eqs. (4) apply to the problem studied in this paper.

Two forms of Eqs. (4) are particularly useful. Both reduce the order of the equations by factoring out the translational degree of freedom represented by the action of $T_{\phi}:\left(z_{1}, z_{2}\right) \rightarrow\left(e^{i \phi} z_{1}, e^{2 i \phi} z_{2}\right), \phi \equiv$ $\ell / k$, corresponding to spatial translations $x \rightarrow x+\ell$. The "polar" coordinates defined by $z_{j}=a_{j} e^{i \phi_{j}}$ and the $T_{\phi}$-invariant relative phase $\theta=\phi_{2}-2 \phi_{1}$ are standard:

$$
\begin{aligned}
\dot{a}_{1} & =\left(\mu_{1}+d_{11} a_{1}^{2}+d_{12} a_{2}^{2}\right) a_{1}+a_{1} a_{2} \cos \theta, \\
\dot{a}_{2} & =\left(\mu_{2}+d_{21} a_{1}^{2}+d_{22} a_{2}^{2}\right) a_{2}+\sigma a_{1}^{2} \cos \theta, \\
\dot{\theta} & =-\frac{1}{a_{2}}\left(\sigma a_{1}^{2}+2 a_{2}^{2}\right) \sin \theta .
\end{aligned}
$$

The evolution of the individual phases $\phi_{j}$ is slaved to that of $a_{1}, a_{2}$ and $\theta$, and is given by

$$
\dot{\phi}_{1}=a_{2} \sin \theta, \quad \dot{\phi}_{2}=-\sigma \frac{a_{1}^{2}}{a_{2}} \sin \theta .
$$

Thus fixed points of the $\theta$ equation with $\dot{\phi}_{j} \neq 0$ correspond to periodic solutions of equations (4) while limit cycles in (5) correspond to tori. In the following we refer to (nontrivial) solutions with $\dot{\phi}_{1}=\dot{\phi}_{2}=0$ as standing; such solutions are necessarily reflection-symmetric and in the variables (5) they are found in the union of the two subspaces defined by $\cos \theta= \pm 1$.
The second and more useful form of Eqs. (4) is obtained from (5) on using the variables $x=$ $a_{2} \cos \theta, y=a_{2} \sin \theta$ :

$$
\begin{aligned}
\dot{a}_{1}= & \left(\mu_{1}+d_{11} a_{1}^{2}+d_{12}\left(x^{2}+y^{2}\right)\right) a_{1}+a_{1} x, \\
\dot{x}= & \left(\mu_{2}+d_{21} a_{1}^{2}+d_{22}\left(x^{2}+y^{2}\right)\right) x \\
& +2 y^{2}+\sigma a_{1}^{2}, \\
\dot{y}= & \left(\mu_{2}+d_{21} a_{1}^{2}+d_{22}\left(x^{2}+y^{2}\right)\right) y-2 x y .
\end{aligned}
$$

In this representation the standing solutions are contained within a single invariant plane, $y=0$, and due to the invariance of (6c) under $y \rightarrow-y$ (corresponding to invariance under spatial reflection) we can, without loss of generality, restrict attention to solutions with $y \geq 0$, i.e. to $\theta \in[0, \pi]$. All solutions with $y>0$ and $a_{1}>0$ represent drifting states. Figure 19(a) shows the various relevant structures in the $\left(a_{1}, x, y\right)$ space. There are two types of fixed points, both in the plane $y=0$, the $n=1$ mixed mode (characterized by $a_{1} x>0$ and labeled $M M_{0}$ ), and two possible $n=2$ pure modes (characterized by $a_{1}=0$ ) whose phase relative to the $n=1$ state is either 0 or $\pi$; these are called $P_{0}$, and $P_{\pi}$, respectively. Within the system (4) these states are obtained from one another by a spatial translation by $L / 4$, and hence have identical eigenvalues. The same symmetry, i.e. the invariance of Eqs. (4) under $\left(z_{1}, z_{2}\right) \rightarrow\left(i z_{1},-z_{2}\right)$, is also responsible for the presence of the structurally stable heteroclinic cycles studied by Armbruster et al. [1988]. Figure 19(a) shows an example. The cycle connects $P_{0}$ to $P_{\pi}$ in the invariant subspace $y=0$, and $P_{\pi}$ back to $P_{0}$ in the pure mode subspace $a_{1}=0$. As already mentioned, the connection $P_{\pi}$ to $P_{0}$ corresponds to

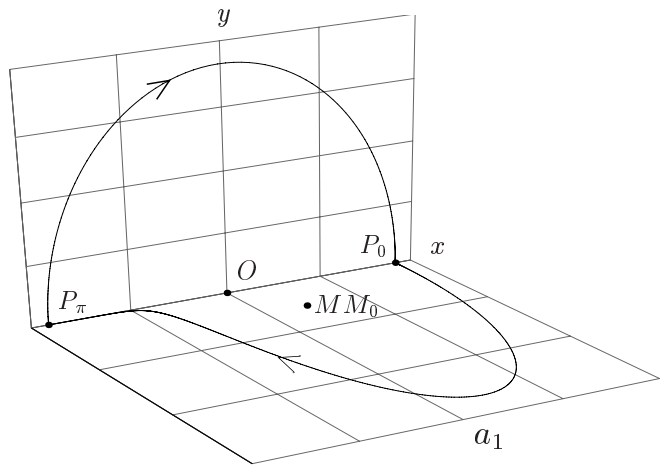

(a)

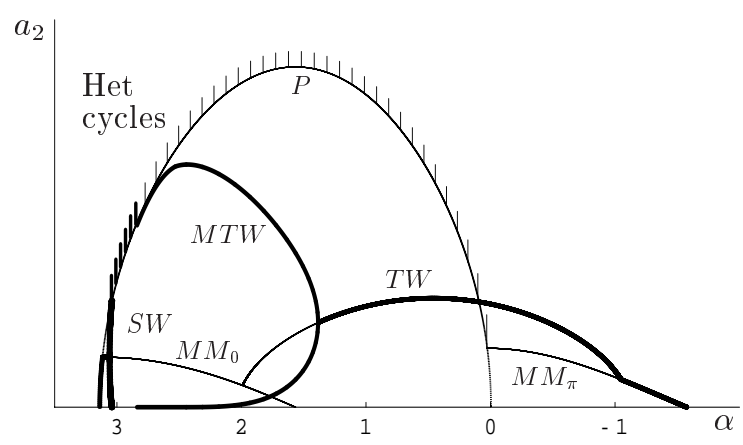

(b)

Fig. 19. (a) The structurally and asymptotically stable AGH cycle in the $\left(a_{1}, x, y\right)$ variables for $\sigma=-1, d_{11}=-0.4$, $d_{12}=1.6, d_{21}=-6, d_{22}=-0.5$ when $|\mu|=0.05$ and $\alpha=2.8$, where $\mu_{1}=|\mu| \cos \alpha, \mu_{2}=|\mu| \sin \alpha$. (b) The corresponding bifurcation diagram with $\alpha$ as the bifurcation parameter. After Porter and Knobloch [2001]. 
a change in the phase $\phi_{1}$ by $\pi / 2$ while the system stays in a fixed $n=2$ state. Since both subspaces are two-dimensional both connections persist under changes in the parameters $\mu_{1}$ and $\mu_{2}$. For other parameter values the fixed point $M M_{0}$ may be absent, or undergo a Hopf bifurcation in the $y=0$ plane creating an unstable limit cycle (SW).

In Fig. 19(a) the fixed point $M M_{0}$ is repelling in the $y=0$ plane but attracting in the $y$ direction. Figure 19(b) shows how this situation comes about. We write $\left(\mu_{1}, \mu_{2}\right)=|\mu|(\cos \alpha, \sin \alpha)$ and decrease $\alpha$ along $|\mu|=0.05$. The fixed point $M M_{0}$ is initially attracting, but soon undergoes a supercritical Hopf bifurcation that produces a branch of stable standing waves. As $\alpha$ decreases further the amplitude of these standing oscillations grows and a heteroclinic connection between the origin and $P_{0}$ forms, i.e. the unstable manifold of $P_{0}$ connects to the origin. When $\alpha$ is decreased this connection breaks and the unstable manifold of $P_{0}$ now connects to $P_{\pi}$, forming the heteroclinic cycle depicted in Fig. 19(a). Figures 17 and 18 suggest that this is exactly what is happening in the pdes $(2 \mathrm{a})-(2 \mathrm{e})$ when $\sigma=0.1$, and $B_{+}^{*}=1, B_{-}^{*}=0$. Figure $19(\mathrm{~b})$ shows that this heteroclinic cycle exists over a wide range of $\alpha$ but that it loses stability with decreasing $\alpha$ at the point where the modulated traveling waves collide with the circle of pure modes $n=2$. At this point the cycle transfers its stability (without hysteresis) to the MW and vice versa. In our numerical solutions of the pdes (2a)-(2e) we find evidence for a global bifurcation of this kind as well (see Sec. 3.1). Moreover, an examination of the structure of Eqs. (4) suggests that if one starts with an initial condition $\left(z_{1}, 0\right)$ with $\operatorname{Im} z_{1}=0$ the system will evolve in the invariant subspace $\operatorname{Im} z_{1}=\operatorname{Im} z_{2}=0$, and similarly for an initial condition $\left(z_{1}, 0\right)$ with $\operatorname{Re} z_{1}=0$. However, it is simple to see that Eqs. (4) restricted to either of these invariant subspaces $[y=0$ in Eqs. (6)] do not describe the switching observed in the pdes: the switching only occurs if $\dot{\theta} \neq 0$, however briefly, a fact consistent with our computation of the equantity $\arg \theta_{2}-2 \arg \theta_{1}$ in Sec. 3.1. These considerations suggest that it is the proximity to such invariant subspaces that distinguishes between periodic and nonperiodic switching. If the system is started exceedingly close to an invariant subspace it will exhibit switching, but its dynamics will be lower-dimensional, and consequently complex behavior (beyond the formation of the structurally stable connection between $P_{0}$ and $P_{\pi}$ ) is less likely. In contrast, with generic initial conditions the system never approaches an invariant subspace quite so closely and its dynamical behavior is therefore less constrained. Whether this is a consequence of round-off error is difficult to tell [Berkooz et al., 1994], but all our tests suggest that it may be the higher modes that are present in the pdes that prevent the formation of the structurally stable heteroclinic cycle (or perhaps destabilize it), with the result that a long periodic orbit is present instead [Fig. 10(a)].

In this article we have shown that the loss of midplane reflection symmetry may have profound dynamical consequences even in two-dimensional convection. We focused on the consequences of heat loss through the upper boundary, or of heating the system at constant electrical power instead of fixed temperature, both effects measured by effective Biot numbers. The inclusion of these physically important effects necessitates a reformulation of the problem in terms of a modified Rayleigh number $\mathrm{Ra}^{\prime}$ defined in such a way that $\mathrm{Ra}^{\prime}$ remains constant for fixed values of the externally imposed parameters. This is because, as shown in Figs. 18 and 14, the conventionally defined Rayleigh number Ra [Eq. (1c)] fluctuates in time, much as the Nusselt number or the mean flow associated with drifting states; in contrast the modified Rayleigh number $\mathrm{Ra}^{\prime}$ employed here remains constant. The resulting reformulation should be of substantial help in quantitative comparisons between experiments and theory. We have noted that near the $1: 2$ spatial resonance the resulting loss of midplane reflection symmetry creates intervals in $\mathrm{Ra}^{\prime}$ containing no stable steady or steadily drifting states. The bifurcation diagram that results bears a substantial resemblance to that of the Kuramoto-Sivashinsky equation [Armbruster et al., 1989] except for the fact that in the latter the Hopf bifurcation to the modulated traveling waves is subcritical. In these intervals we showed, by direct numerical simulation, that the system settles into an almost heteroclinic cycle connecting two pure modes related by translation by $L / 4$; this cycle may be periodic with a long period, or chaotic with a long mean switching time, depending on the initial conditions. Generic initial conditions lead to complex dynamics, but the dynamics become much simpler (and periodic) for initial conditions sufficiently near reflection-invariant subspaces. Similar behavior is obtained in the stability gaps created by homotopic continuation between no-slip and stress-free boundary conditions at the top (cf. Fig. 9(a) of Prat et al. [2002]). 
These results are consistent with those of a related study of the (simpler) Kuramoto-Sivashinsky equation [Armbruster et al., 1989; Kevrekidis et al., 1990] for which the presence of structurally stable connections between $n=2$ states and their $L / 4$ translates was established using reduction to normal form [Armbruster et al., 1989] and careful numerics on a Galerkin expansion of the equation [Kevrekidis et al., 1990]. As in the present case numerical simulations of the Kuramoto-Sivashinsky equation reported by Kevrekidis et al. revealed periodic or nearly periodic switching between an $n=2$ state and its $L / 4$ translate, although this observation could be a consequence of the use of Fast Fourier Transforms (i.e. of numerical error). Moreover, no chaotic switching was reported. As already mentioned we have checked our results using two independent codes, and believe them to be correct. Both codes preserve exactly the symmetries of the pdes since they employ Fourier decomposition in $x$. However, the results obtained superficially conflict with our independent determination of the eigenvalue ratio $\rho$ (Fig. 8). The fact that $\rho>1$ for $\mathrm{Ra}^{\prime}>1863$ implies that a heteroclinic cycle connecting an $n=2$ fixed point with its $L / 4$ translate (if present) will be asymptotically stable for $\mathrm{Ra}^{\prime}>1863$ and hence should attract all trajectories starting near but not necessarily in the invariant subspace. The observation of chaotic switching for initial conditions that are not in the invariant subspace conflicts with this statement. Thus, while the possibility remains that both the periodic and chaotic switching reported above is in fact due to numerical error, the possibility that the higher order modes prevent the formation of the structurally stable heteroclinic cycle, is also worth considering. The presence of such modes raises the dimension of the stable manifold of the fixed points, and hence a trajectory starting from one fixed point is no longer forced to connect to its translate, in contrast to the situation in the normal form (4). In such circumstances any connections that might be present need not persist under changes of $\mathrm{Ra}^{\prime}$. The papers of Kevrekidis et al. [1990] on the KuramotoSivashinsky equation, and of Aubry et al. [1988], and Sanghi and Aubry [1993] on models of the turbulent boundary layer, provide some information about the possible role of higher order modes. Kevrekidis et al. [1990] showed, using careful numerics on a Galerkin truncation, that for their problem the connection persists, but their FFT simulations reveal periodic (or nearly periodic) switching.
In contrast Aubry et al. pointed out that near the global bifurcation at which a putative heteroclinic cycle acquires stability the switching interval will increase only very slowly with time (by a factor of approximately $\rho^{2}$ each time around the cycle) but that further away it should increase much more rapidly since $\rho$ becomes larger. They compare these predictions with numerical integration of a 10 variable (real) system and find that although the switching interval does increase with time in all cases, the rate of increase is typically substantially slower than the theoretical prediction, an effect they attribute to rounding error. However, in no case do they find that the switching interval actually saturates, in contrast to both the Kuramoto-Sivashinsky simulations and the results reported above. On the other hand they also emphasize that the addition of small random perturbations arising from such errors may produce a "statistical limit cycle", as already suggested by Busse [1981], and the switching interval would then saturate. For the purposes of the present paper it is also important to note that even for this relatively low-dimensional system Aubry et al. are unable to prove the existence of the required heteroclinic cycle. An actual pde or, as here, a system of pdes is of course equivalent to an ode system of much higher dimension, even when integrated numerically. In contrast, Campbell and Holmes [1991] showed that the presence of higher order modes may allow the pure modes themselves to lose stability via a parity-breaking bifurcation, and suggest that if such a bifurcation occurs in the heteroclinic regime, the system behavior will resemble that of a heteroclinic cycle but with a superposed drift. This situation does not apparently arise in the present problem. Regrettably, aside from the work of Armbruster et al. [1989] on the KuramotoSivashinsky equation and the one-dimensional example studied by Cox [1996], we know of no other work that attempts a detailed comparison between the normal form results and direct numerical simulations of pdes, and hence of additional results pertaining to the structural stability of the dynamics in the $1: 2$ spatial resonance with respect to the inclusion of additional modes. The example in the present paper suggests that the structurally stable heteroclinic cycles are in fact quite fragile, most likely due to the presence of $n=3$ modes, even though they do leave behind echos of their dynamics, a conclusion that conforms to that of Aubry et al. 
It remains to consider the mechanism whereby the structurally stable heteroclinic cycles disappear as $\mathrm{Ra}^{\prime}$ continues to increase. In the normal form this occurs when a standing wave (SW) in the reflectioninvariant subspace forms a heteroclinic connection between the origin and the $n=2$ fixed points. In case I of Porter and Knobloch [2001] and for sufficiently small $|\mu|$ the SW are stable, and the heteroclinic cycle is replaced by stable standing oscillations as $\alpha$ increases (Fig. 19). In the case studied by Aubry et al. and for larger values of $|\mu|$ in the cases studied by Porter and Knobloch the SW are unstable. In these circumstances the system undergoes instead a hysteretic transition to the $n=1$ state and all oscillations cease. In the example studied in the present paper neither of these terminations is possible. This is because no $n=1$ states (and no standing waves) are present beyond $\mathrm{Ra}^{\prime}=1840.1$. Instead it appears that in our case the cycles disappear at $\mathrm{Ra}^{\prime} \approx 1935$ where an $n=1$ mode bifurcates from the primary $n=2$ branch in a subcritical steady state bifurcation. Cox [1996] has found similar behavior in a long wave equation that applies in the limit $B_{-}^{*}=B_{+}^{*}=0$ (i.e. for insulating boundaries) with different velocity boundary conditions at the top and bottom.

Whether similar behavior occurs in other physical systems under realistic conditions remains unclear, although the work of Chossat [2001] suggests that it should be a general property of hydrodynamic systems. Jones [1982] has computed a diagram similar to our Fig. 3 for axisymmetric Taylor vortices with axial wavenumbers in the $1: 2$ ratio, but found no evidence of a secondary paritybreaking bifurcation. Proctor and Jones [1988] analyzed a two-layer system using the normal form (4), locating parameter regimes with structurally stable heteroclinic cycles, but did not study the dynamics in the original pdes. Perhaps the best system for further study of the dynamical behavior studied here is provided by the Bénard-Marangoni problem in a cylinder [Echebarría et al., 1997] where heteroclinic cycles were also identified. Simulations of the relevant pdes as well as further experiments on this system are therefore of great interest.

Added note: Recent work by C. Nore et al. [2001] identifies a $1: 2$ spatial resonance in the von Kármán flow between counter-rotating disks and obtains results closely related to ours.

\section{Acknowledgments}

This work was supported in part by DGESIC under grant PB97-0683 (I. Moroz and J. Prat) and NSF under grant DMS-0072444 (E. Knobloch). We thank the Fulbright Foundation for additional support. We are grateful to J. Porter for a careful reading of the manuscript, to C. Nore and L. Tuckerman for communicating to us their results prior to publication, and to S. Cox for helpful discussions.

\section{References}

Armbruster, D. [1987] "O(2)-symmetric bifurcation theory for convection rolls," Physica D27, 433-439.

Armbruster, D., Guckenheimer, J. \& Holmes, P. [1988] "Heteroclinic cycles and modulated travelling waves in systems with $\mathrm{O}(2)$ symmetry," Physica D29, 257-282.

Armbruster, D., Guckenheimer, J. \& Holmes, P. [1989] "Kuramoto-Sivashinsky dynamics on the center-unstable manifold," SIAM J. Appl. Math. 49, 676-691.

Aubry, N., Holmes, P., Lumley, J. L. \& Stone, E. [1988] "The dynamics of coherent structures in the wall region of a turbulent boundary layer," J. Fluid Mech. 192, 115-173.

Berkooz, G., Holmes, P., Lumley, J. L., Aubry, N. \& Stone, E. [1994] "Observation regarding 'Coherence and chaos in a model of turbulent boundary layer' by X. Zhou and L. Sirovich [Phys. Fluids A4, 2855 (1992)]," Phys. Fluids 6, 1574-1578.

Busse, F. H. [1981] "Transition to turbulence in Rayleigh-Bénard convection," in Hydrodynamic Instabilities and the Transition to Turbulence, eds. Swinney, H. L. \& Gollub, J. P. (Springer-Verlag), pp. $97-137$.

Campbell, S. A. \& Holmes, P. [1991] "Bifurcation from $\mathrm{O}(2)$ symmetric heteroclinic cycles with three interacting modes," Nonlinearity 4, 697-726.

Chossat, P. [2001] "The bifurcation of heteroclinic cycles in systems of hydrodynamic type," Dyn. Contin. Discr. Impul. Syst., Ser. A. Math. Anal. 8, 575-590.

Clune, T. L. [1993] "Pattern selection in convecting systems," Ph.D. Thesis, University of California at Berkeley.

Cox, S. M. [1996] "Mode interactions in Rayleigh-Bénard convection," Physica D95, 50-61.

Dangelmayr, G. [1986] "Steady-state mode interactions in the presence of O(2)-symmetry," Dyn. Stab. Syst. 1, 159-185.

Deane, A. E., Knobloch, E. \& Toomre, J. [1987] "Traveling waves and chaos in thermosolutal convection," Phys. Rev. A36, 2862-2869.

Drissi, A., Net, M. \& Mercader, I. [1999] "Subharmonic instabilities of Tollmien-Schlichting waves in 
two-dimensional Poiseuille flow," Phys. Rev. E60, 1781-1791.

Echebarría, B., Krmpotić, D. \& Pérez-García, C. [1997] "Resonant interactions in Bénard-Marangoni convection in cylindrical containers," Physica D99, 487-502.

Holmes, P., Lumley, J. L. \& Berkooz, G. [1996] Turbulence, Coherent Structures, Dynamical Systems and Symmetry (Cambridge University Press, Cambridge).

Jones, C. A. [1982] "On the flow between counterrotating cylinders," J. Fluid Mech. 120, 433-450.

Karniadakis, G. E., Israeli, M. \& Orszag, S. A. [1991] "High-order splitting methods for the incompressible Navier-Stokes equations," J. Comput. Phys. 97, 414-443.

Kevrekidis, I. G., Nicolaenko, B. \& Scovel, J. C. [1990] "Back in the saddle again: A computer assisted study of the Kuramoto-Sivashinsky equation," SIAM J. Appl. Math. 50, 760-790.

Knobloch, E. [1990] "Pattern selection in longwavelength convection," Physica D41, 450-479.

Krupa, M. \& Melbourne, I. [1995] "Asymptotic stability of heteroclinic cycles in systems with symmetry," Ergod. Theor. Dynam. Syst. 15, 121-147.

Manogg, G. \& Metzener, P. [1994] "Strong resonance in two-dimensional non-Boussinesq convection," Phys. Fluids 6, 2944-2955.

Mercader, I., Prat, J. \& Knobloch, E. [2001] "The 1:2 mode interaction in Rayleigh-Bénard convection with weakly broken midplane symmetry," Int. J. Bifurcation and Chaos 11, 27-41.

Nore, C., Tuckerman, L., Daube, O. \& Xin, S. [2001] "The $1: 2$ mode interaction in exactly counter-rotating von Kármán swirling flow," J. Fluid Mech., in press.

Petrescu, C.-D. [1998] "Sélection des grandes structures horizontal de la convection de Rayleigh-Bénard," Ph.D. Thesis, Ecole Polytechnique Fédérale de Lausanne.

Pismen, L. M. [1986] "Inertial effects in long-scale thermal convection," Phys. Lett. A116, 241-244.

Platten, J. K. \& Legros, J. C. [1984] Convection in Liquids (Springer-Verlag, Berlin).

Pontes, J., Christov, C. I. \& Velarde, M. G. [1996] "Numerical study of patterns and their evolution in finite geometries," Int. J. Bifurcation and Chaos 6, 1883-1890.

Pontes, J., Christov, C. I. \& Velarde, M. G. [1999] "Numerical approach to pattern selection in a model problem for Bénard convection in a finite fluid layer," $A n$ nuaire de l'Université de Sofia "St. Kliment Ohridski" 93, 157-175.
Porter, J. \& Knobloch, E. [2000] "Complex dynamics in the 1:3 spatial resonance," Physica D143, 138-168.

Porter, J. \& Knobloch, E. [2001] "New type of complex dynamics in the $1: 2$ spatial resonance," Physica D159, 125-154.

Prat, J., Massaguer, J. M. \& Mercader, I. [1995] "Largescale flows and resonances in 2-D thermal convection," Phys. Fluids 7, 121-134.

Prat, J., Mercader, I. \& Knobloch, E. [1998] "Resonant mode interaction in Rayleigh-Bénard convection," Phys. Rev. E58, 3145-3156.

Prat, J., Mercader, I. \& Knobloch, E. [2001] "RayleighBénard convection with experimental boundary conditions," Proc. Conf. Bifurcations, Symmetry and Patterns (Birkhäuser-Verlag), in press.

Prat, J., Mercader, I. \& Knobloch, E. [2002] "The 1:2 mode interaction in Rayleigh-Bénard convection with and without Boussinesq symmetry," Int. J. Bifurcation and Chaos 12, 281-308.

Proctor, M. R. E. [1981] "Planform selection by finiteamplitude thermal convection between poorly conducting slabs," J. Fluid Mech. 113, 469-485.

Proctor, M. R. E. \& Jones, C. A. [1988] "The interaction of two spatially resonant patterns in thermal convection. Part 1. Exact 1:2 resonance," J. Fluid Mech. 188, 301-335.

Recktenwald, A. \& Lücke, M. [1998] "Thermoconvection in magnetized ferrofluids: The influence of boundaries with finite heat conductivity," J. Magn. Magn. Mater. 188, 326-332.

Sanghi, S. \& Aubry, N. [1993] "Mode interaction models for near-wall turbulence," J. Fluid Mech. 247, 455-488.

Steinberg, V., Fineberg, J., Moses, E. \& Rehberg, I. [1989] "Pattern selection and transition to turbulence in propagating waves," Physica D37, 359-383.

Sullivan, T. S. \& Ahlers, G. [1988] "Hopf bifurcation to convection near the codimension-two point in a ${ }^{3} \mathrm{He}-$ ${ }^{4}$ He mixture," Phys. Rev. Lett. 61, 78-81.

Surko, C. M., Kolodner, P., Passner, A. \& Walden, R. W. [1986] "Finite-amplitude traveling-wave convection in binary fluid mixtures," Physica D23, 220-229.

Villers, D. \& Platten, J. K. [1984] "Heating curves in the two-component Bénard problem," J. Non-Equilib. Thermodyn. 9, 131-146.

Zhou, X. \& Sirovich, L. [1992] "Coherence and chaos in a model of turbulent boundary layer," Phys. Fluids A4, 2855-2874. 